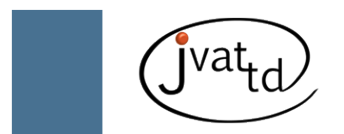

\title{
Checklist of helminths from lizards and amphisbaenians (Reptilia, Squamata) of South America
}

Ávila RW (1), Silva RJ (1)

(1) Department of Parasitology, Botucatu Biosciences Institute, São Paulo State University (UNESP - Univ Estadual Paulista), Botucatu, São Paulo State, Brazil.

Abstract: A comprehensive and up to date summary of the literature on the helminth parasites of lizards and amphisbaenians from South America is herein presented. One-hundred eighteen lizard species from twelve countries were reported in the literature harboring a total of 155 helminth species, being none acanthocephalans, 15 cestodes, 20 trematodes and 111 nematodes. Of these, one record was from Chile and French Guiana, three from Colombia, three from Uruguay, eight from Bolivia, nine from Surinam, 13 from Paraguay, 12 from Venezuela, 27 from Ecuador, 17 from Argentina, 39 from Peru and 103 from Brazil. The present list provides host, geographical distribution (with the respective biome, when possible), site of infection and references from the parasites. A systematic parasite-host list is also provided.

Key words: Cestoda, Nematoda, Trematoda, Squamata, neotropical.

\section{INTRODUCTION}

Parasitological studies on helminths that infect squamates (particularly lizards) in South America had recent increased in the past few years, with many new records of hosts and/or localities and description of several new species (1-3). Such studies, however, date back to 1920, when Dr. Lauro Travassos and his colleagues presented important contributions to the field of helminth systematics (4-6).

The first comprehensive efforts to summarize the knowledge about helminths of squamates are those from Baker (7), who listed all available published data on the occurrence of nematodes infecting reptiles and amphibians. Other studies have a regional scope and treat each helminth group separately, for example, nematodes collected from reptiles of Brazil, digenetic trematodes of amphibians and reptiles from Argentina and acanthocephalans in amphibians and reptiles from Brazil and Paraguay (8-10).
The present checklist summarizes the diversity of helminths from lizards and amphisbaenians of South America, providing a host-parasite list with localities and biomes.

\section{STUDIED REGIONS}

This checklist was prepared based on records of nematodes, acanthocephalans, cestodes, and trematodes published from 1914 to 2010, excluding material from the so called "grey literature", that is, records of unpublished theses or scientific meetings. The taxonomy of helminths follows: nematodes, cestodes, trematodes and acanthocephalans (6, 8, 11-15). Whenever possible, taxonomy of hosts is reviewed with recent publications.

Each record is constituted by the class, order, superfamily, family and species of the helminth; as well as the name of the authority who described it and year; besides its host(s), geographical record with country and state/province (when available) and information on biome of host. Comments 
include taxonomy, synonyms and also changes in the host taxonomy.

Abbreviations for all political units cited in this paper are:

- Brazil: Acre (AC), Alagoas (AL), Amapá (AP), Amazonas (AM), Bahia (BA), Ceará (CE), Distrito Federal (DF), Goiás (GO), Espírito Santo (ES), Maranhão (MA), Mato Grosso (MT), Mato Grosso do Sul (MS), Minas Gerais (MG), Pará (PA), Paraíba (PB), Paraná (PR), Pernambuco (PE), Piauí (PI), Rio de Janeiro (RJ), Rio Grande do Norte (RN), Rio Grande do Sul (RS), Rondônia (RO), Roraima (RR), São Paulo (SP), Santa Catarina (SC), Sergipe (SE), and Tocantins (TO).

- Argentina: Buenos Aires (BA), Catamarca (CA), Córdoba (CR), Corrientes (CO), Chaco $(\mathrm{CH})$, Chubut $(\mathrm{CB})$, Entre Rios (ER), Formosa (FO), Jujuy (JU), La Pampa (LP), La Rioja (LR), Mendoza (ME), Misiones (MI), Neuquén (NE), Rio Negro (RN), Salta (SA), San Juan (SJ), San Luis (SL), Santa Cruz (SC), Santa Fe (SF), Santiago del Estero (SE), Tierra del Fuego (TF), and Tucumán (TU).

- Paraguay: Alto Paraguay (AP), Alto Paraná (AA), Amambay (AM), Distrito Capital (DC), Boquerón (BO), Caaguazú (CA), Caazapá (CZ), Canindeyú (CN), Central (CE), Concepción (CO), Cordillera (CR), Guairá (GU), Itapúa (IT), Misiones (MI), Ñeembucú (NE), Paraguarí (PA), Presidente Hayes (PH), and San Pedro (SP).

- Bolivia: Chuquisaca $(\mathrm{CH})$, Cochabamba (CO), Beni (BE), La Paz (LP), Oruro (OR), Pando (PA), Potosí (PO), Santa Cruz (SC), and Tarija (TA).

- Venezuela: Amazonas (AM), Anzoátegui (AN), Apure (AP), Aragua (AR), Barinas (BA), Bolívar (BO), Carabobo (CA), Cojedes (CO), Delta Amacuro (DA), Falcón (FA), Guárico (GU), Lara (LA), Mérida (ME), Miranda (MI), Monagas (MO), Nueva Esparta (NE), Portuguesa (PO), Sucre (SU), Táchira (TA), Trujillo (TR), Yaracuy (YA), Vargas (VA), and Zulia (ZU).

- Ecuador: Azuay (AZ), Bolívar (BO), Cañar (CA), Carchi (CR), Cotopaxi (CO), Chimborazo (CH), Galápagos (GA), Imbabura (IM), Loja (LO), Pichincha (PI), Tungurahua (TU), El Oro (EO), Esmeraldas (ES), Guayas (GU), Los Ríos (LR), Manabí (MA), MoronaSantiago (MS), Napo (NA), Pastaza (PA),
Zamora-Chinchipe (ZC), Sucumbíos (SU), and Orellana (OR).

- Peru: Amazonas (AM), Ancash (AN), Apurímac (AP), Arequipa (AR), Ayacucho (AY), Cajamarca (CA), Callao (CL), Cusco (CU), Huancavelica (HU), Huánuco (HA), Ica (IC), Junin (JU), La Libertad (LL), Lambayeque (LA), Lima (LI), Loreto (LO), Madre de Dios (MD), Moquegua (MO), Pasco (PA), Piura (PI), Puno (PU), San Martín (SM), Tacna (TA), Tumbes (TU), and Ucayali (UC).

- Colombia: Amazonas (AM), Antioquia (AN), Arauca (AR), Atlántico (AT), Bolívar (BO), Boyacá (BY), Caldas (CA), Caquetá (CQ), Casanare (CS), Cauca (CU), Cesar (CE), Chocó $(\mathrm{CH})$, Córdoba (CO), Cundinamarca $(\mathrm{CN})$, Guainía (GU), Guaviare (GA), Huila (HU), La Guajira (LG), Magdalena (MA), Meta (ME), Nariño (NA), Norte de Santander (NS), Putumayo (PU), Quindío (QU), Risaralda (RI), San Andrés (SA), Santander (SN), Sucre (SU), Tolima (TO), Valle del Cauca (VC), Vaupés (VA), and Vichada (VI).

- Chile: Aisén (AI), Antofagasta (AN), Araucanía (AU), Arica (AR), Atacama (AT), Biobío (BI), Coquimbo (CO), Los Rios (LR), Los Lagos (LL), Magallanes (MG), Maule (MA), O'Higgins (OH), Santiago (AS), Tarapacá (TA) and Valparaiso (VA).

- Uruguay: Artigas (AR), Canelones (CA), Cerro Largo (CL), Colônia (CO), Durazno (DU), Flores (FL), Florida (FO), Lavalleja (LA), Maldonado (MA), Montevidéu (MO), Paysandú (PA), Río Negro (RN), Rivera (RI), Rocha (RO), Salto (SA), San José (SJ), Soriano (SO), Tacuarembó (TA), and Treinta y Tres (TT).

- Surinam: Brokopondo (BR), Commewijne (CO), Coronie (CR), Marowijne (MA), Nickerie (NI), Para (PA), Paramaribo (PR), Saramacca (SA), Sipaliwini (SI), and Wanica (WA).

- French Guiana: Awala-Yalimapo (AY), Apatou (AP), Camopi (CA), Cayenne (CY), Grand-Santi (GS), Iracoubo (IR), Kourou (KO), Macouria (MC), Mana (MA), Maripasoula (MR), Matoury (MT), Montsinéry-Tonnegrande (MO), Ouanary (OU), Papaïchton (PA), Régina (RE), RemireMontjoly (RM), Roura (RO), Saint-Élie (SE), Saint-Georges (SG), Saint-Laurent-du-Maroni (SL), Saül (SA), and Sinnamary (SI). 
- Guyana: Barima-Waini (BW), PomeroonSupenaam (PS), Essequibo Islands-West Demerara (EI), Demerara-Mahaica (DM), Mahaica-Berbice (MB), East BerbiceCorentyne (EC), Cuyuni-Mazaruni (CM), Potaro-Siparuni (PS), Upper Takutu-Upper Essequibo (TE), and Upper Demerara-Berbice (DB).

\section{LIST OF HELMINTHS}

\section{Acanthocephala}

Hosts: Tropidurus torquatus Wied-Neuwied, 1820 Site of infection: Stomach wall

Distribution: Argentina - CO (Chaco).

Comments: No indication of family and/or genus, but probably belong to Centrorhynchidae, since several cystacanths of this family have been found in South American lizards (16).

Archiacanthocephala

Oligacanthorhynchida

Oligacanthorhynchidae

Oligacanthorhynchus Travassos, 1915

Oligacanthorhynchus sp.

Hosts: Ameiva ameiva Linnaeus, 1758

Distribution: Paraguay - Rio Negro (Chaco), PH (10).

\section{Palaeacanthocephala}

Echinorhynchida

\section{Echinorhynchidae}

Hosts: Mabuya macrorhyncha Hoge, 1946

Site of infection: Stomach wall

Distribution: Brazil - Trancoso (Restinga), BA.

Comments: No indication of genus (17).

\section{Acanthocephalus Koelreuther, 1771}

Acanthocephalus saurius Bursey \& Goldberg, 2003

Hosts: Cercosaura oshaugnessyi Boulenger, 1885

Site of infection: Small intestine

Distribution: Brazil - AC (Amazon) (18).

Acanthocephalus sp.

Hosts: Enyalius perditus Jackson, 1978

Site of infection: Stomach

Distribution: Brazil - São Sebastião (Atlantic

Forest), SP (3).

Echinorhynchus Zoega in Müller, 1776

Echinorhynchus sp.
Hosts: Tropidurus torquatus

Site of infection: Small intestine

Distribution: Brazil (19).

\section{Polymorphida}

\section{Centrorhynchidae}

Hosts: Mabuya macrorhyncha; M. agilis Raddi, 1823, Hemidactylus mabouia Moreau de Jonnés, 1818, Enyalius bilineatus Duméril \& Bibron, 1837

Site of infection: Peritonium, liver; mesentery Distribution: Brazil - Queimada Grande island (Atlantic forest) and Valinhos (Cerrado), SP; Grussaí (Restinga) and Nova Iguaçu (Atlantic Forest), RJ; Praia das Neves (Restinga) and Marechal Floriano (Atlantic Forest), ES.

Comments: All citations above consist of cystacanth occurrence and are, thus, difficult to identify to species level, because reproductive structures are required (20-24).

\section{Centrorhynchus Lühe, 1911}

Centrorhynchus tumidulus Rudolphi, 1919

Hosts: Ameiva ameiva; Tropidurus torquatus; Tupinambis teguixin Linnaeus, 1758

Site of infection: Body cavity

Comments: Tupinambis teguixin found in Rio de Janeiro and in open habitats in South America may actually correspond to T. merianae.

Distribution: Brazil - Rio de Janeiro (Atlantic Forest), RJ $(25,26)$.

Centrorhynchus sp.

Hosts: Tupinambis teguixin

Site of infection: Not informed

Distribution: Paraguay - AP (Chaco) (10).

\section{Nematoda}

Adenophorea

Enoplida

Capilariidae

Capillaria (Zeder, 1800) Bell \& Beverley Burton 1981

Capillaria (= Amphibiocapillaria) freitaslenti Araujo \& Gandra, 1941

Hosts: Tropidurus torquatus; Ameiva ameiva

Site of infection: Intestine

Distribution: Brazil - GO (Cerrado); Peru - CU (Amazon) $(1,8)$.

Ichthyocapillaria Moravec, 1982

Ichthyocapillaria (= Pseudocapillaria) maricaensis 
Rodrigues, 1992

Hosts: Liolaemus lutzae Mertens, 1938

Site of infection: Small intestine

Distribution: Brazil - Maricá (Restinga), RJ (27).

\section{Ascaridida}

Anisakidae

\section{Contracaecum Railliet \& Henry, 1912}

Contracaecum sp.

Hosts: Tupinambis teguixin

Site of infection: Not informed

Distribution: Surinam - PR (Amazon) (28).

\section{Ascarididae}

Dujardinascaris Baylis, 1927

Dujardinascaris sp.

Hosts: Kentropyx pelviceps Cope, 1868,

Tupinambis teguixin

Site of infection: Stomach

Distribution: Peru - CU (Amazon) (1).

\section{Freitasascaris Sprent, 1983}

Freitasascaris alata Baylis, 1947

Hosts: Tupinambis teguixin

Site of infection: Intestine

Distribution: Surinam - PR (Amazon); Brazil -

AM (Amazon); Uruguay - MO

Comments: Tupinambis teguixin from Uruguay may actually correspond to T. merianae. (28-30).

\section{Hexametra Travassos, 1919}

Hexametra boddaertii Baird, 1860

Hosts: Cnemidophorus littoralis Rocha, Araujo, Vrcibradic \& Costa, 2000; C. ocellifer Spix, 1825; Mabuya agilis; M. frenata Cope, 1862; $M$. macrorhyncha; Tropidurus torquatus

Site of infection: Body cavity

Distribution: Brazil - Jurubatiba, Grussaí and Marica (Restinga), RJ; Salvador and Trancoso (Restinga), BA; Valinhos (Cerrado), SP (17, 23, 31-35).

\section{Atractidae}

Atractis Dujardin, 1945

Atractis cruciata Linstow, 1902

Hosts: Tupinambis teguixin, Stenocercus roseiventris

Site of infection: Intestine

Distribution: Brazil - Belém (Amazon), PA; Ecuador - PA (Amazon).

Comments: Atractis cruciata is considered a junior synonym of $A$. opeatura $(7,36)$. However, it is considered a valid species by some authors $(8,37-39)$.

\section{Cyrtosomum Gedoelst, 1919}

Cyrtosomum longicaudatum Brenes and Bravo Hollis, 1960

Hosts: Anolis punctatus Daudin, 1802; Anolis transversalis Duméril, 1851

Site of infection: Large intestine

Distribution: Peru - CU (Amazon); Brazil - AM (Amazon) $(1,40)$.

Cyrtosomum sp.

Hosts: Ophiodes striatus

Site of infection: Large intestine

Distribution: Brazil - MS (Cerrado) (41).

\section{Maracaya Díaz-Ungría, 1964}

Maracaya belemensis Adamson \& Baccam, 1988

Hosts: Amphisbaena alba Linnaeus, 1758

Site of infection: Large intestine

Distribution: Brazil - Belém (Amazon), PA (42).

Maracaya graciai Díaz-Ungría, 1969

Hosts: Amphisbaena alba

Site of infection: Intestine

Distribution: Venezuela - Maracay, AR $(7,42)$.

Maracaya pusilla (Miranda, 1924) Adamson \& Baccam, 1988

Hosts: Amphisbaena sp.

Site of infection: Intestine

Distribution: Brazil - BA.

Remarks: The former Aplectana pusilla was transferred to Maracaya (42), according to a revision of the genus Maracaya (43), but it is cited in the genus Aplectana by other authors (42-44).

\section{Cosmocercidae}

gen. sp.

Hosts: Cercosaura argulus Peters, 1863

Site of infection: Small intestine

Distribution: Peru - Rio Nanay (Amazon), LO (45).

\section{Aplectana Railliet \& Henry, 1916}

Aplectana albae Adamson \& Baccam, 1988

Hosts: Amphisbaena alba; Amphisbaena ridleyi

Boulenger, 1890

Site of infection: Intestine

Distribution: Brazil - Belém (Amazon), PA;

Fernando de Noronha Island, PE $(42,46)$. 
Aplectana raillieti Travassos, 1925

Hosts: Amphisbaena alba

Site of infection: Intestine

Distribution: Brazil - Angra dos Reis (Insular Atlantic Forest), RJ.

Comments: The host Amphisbaena fusca was originally cited as Amphisbaena alba $(5,7,8,47)$.

Aplectana tucumanensis Ramallo, Bursey \& Goldberg, 2008

Hosts: Amphisbaena bolivica Mertens, 1929

Site of infection: Large intestine

Distribution: Argentina - San Miguel de Tucumán (Chaco), TU (44).

Aplectana unguiculata Rudolphi, 1819

Hosts: Amphisbaena sp.

Site of infection: Not informed

Distribution: Brazil.

Comments: The species is actually considered as species inquirenda (7).

Aplectana vellardi Travassos, 1926

Hosts: Enyalius perditus

Site of infection: Intestine

Distribution: Brazil - Ibitipoca (Cerrado), MG (48).

\section{Cosmocerca Diesing, 1861}

Cosmocerca rara Freitas \& Vicente, 1966

Hosts: Leposternon microcephalum Wagler, 1824

Site of infection: Large intestine

Distribution: Brazil - Rio de Janeiro (Atlantic forest), RJ $(8,49)$.

Cosmocerca vrcibradici Bursey \& Goldberg, 2004 Hosts: Alopoglossus angulatus Linnaeus, 1758; Alopoglossus atriventris Duellman, 1973; Anolis fuscoauratus D'Orbignyi, 1837; Cercosaura eigenmanni Griffin, 1917; C. oshaugnessyi Boulenger, 1885; Uranoscodon superciliosus Linnaeus, 1758

Site of infection: Intestine

Distribution: Brazil - AC, AM, PA, RO (Amazon); Ecuador - SU (Amazon) (18, 50-52).

\section{Cosmocerca sp.}

Hosts: Enyalius perditus

Site of infection: Stomach

Distribution: Brazil - São Sebastião (Insular Atlantic forest), SP (3).

\section{Paradollfusnema Baker, 1982}

Paradollfusnema amphisbaenia Baker, 1981

Hosts: Leposternon microcephalum

Site of infection: Intestine

Distribution: Brazil - Recife, PE $(8,53)$.

\section{Raillietnema Travassos, 1927}

Raillietnema brachyspiculatum Bursey, Goldberg, Salgado-Maldonado, and Mendez-de-la-Cruz, 1998

Hosts: Morunasaurus annularis (O'Shaughnessy, 1881)

Site of infection: Large intestine

Distribution: Ecuador - PA (Amazon) (39).

\section{Heterakidae}

Africana Travassos, 1920

Africana chabaudi Baker, 1981

Hosts: Uranoscodon superciliosus

Site of infection: Intestine

Distribution: Brazil - PA, RO and RR (Amazon) $(50,54)$.

Africana dardanelosi Ávila \& Silva, 2009

Hosts: Hoplocercus spinosus

Site of infection: Intestine

Distribution: Brazil - MT (Amazon) (55).

\section{Moaciria Freitas, 1956}

Moaciria alvarengai Freitas, 1956

Hosts: Trachylepis atlantica Schmidt, 1945

Site of infection: Large intestine

Distribution: Brazil - Fernando de Noronha Island, $\mathrm{PE}(8,46,56)$.

\section{Spinicauda Travassos, 1920}

Spinicauda spinicauda Olfers, 1819

Hosts: Ameiva ameiva, Tupinambis teguixin, Tupinambis merianae, Trachylepis atlantica, Enyalioides praestabilis (O’Shaughnessey, 1881)

Site of infection: Intestine

Distribution: Brazil - MT (Pantanal), Rio de Janeiro, RJ, São Gonçalo, RN and Fortaleza, CE, Fernando de Noronha Islands, PE; Peru - CU (Amazon); Surinam - PR (Amazon); Venezuela - AR; Ecuador - PA (Amazon) $(4,7,28,39,46$, $50,57-60)$.

\section{Strongyluris Mueller, 1894}

Strongyluris oscari Travassos, 1923

Hosts: Ameiva ameiva; Anolis fuscoauratus; A. 
punctatus; A. transversalis; Enyalius iheringii Boulenger, 1885; E. perditus; Eurolophosaurus nanuzae Rodrigues, 1981; Mabuya agilis; Plica plica Linnaeus, 1758; Plica umbra Linnaeus, 1758; Stenocercus caducus; S. roseiventris D'Orbigny in Duméril \& Bibron 1837; Tropidurus sp.; T. guarani Alvarez, Cei \& Scolaro, 1994; T. spinulosus Cope, 1862; T. torquatus; T. melanopleurus

Site of infection: Stomach, intestine

Distribution: Brazil - AC, AM, BA, CE, DF, ES, GO, MG, MS, PA, PB, RJ, RO, SP (Pantanal, Restinga, Amazon, Caatinga, Atlantic Forest); Argentina (Chaco); Paraguay (Chaco); Ecuador - SU and PA (Amazon); Peru - CU (Amazon); Bolivia - Florida province, SC.

Comments: Currently, 31 species are recognized within the genus, and based on morphological characteristics, only four occurrs in neotropical regions (61). Strongyluris oscari seems to be extremely polymorphic, since the species S. freitasi, S. travassosi and S. sai have been synonimized (62). The records of $S$. oscari infecting T. spinulosus in the region of Salobra, MS, Brazil correspond to T. guarani $(1,3,32,35$, 39-41, 48, 51, 60, 62-69).

\section{Kathlaniidae \\ Cruzia Travassos, 1917}

Cruzia fulleborni Khalil \& Vogelsang, 1930

Hosts: Tupinambis teguixin

Site of infection: Intestine

Distribution: Argentina; Paraguay (7, 70-72).

Cruzia rudolphii Ruiz, 1947

Hosts: Hoplocercus spinosus Fitzinger, 1843

Site of infection: Large intestine

Distribution: Brazil - Dois Irmãos do Buriti (Cerrado), MS (71, 73).

Cruzia tentaculata Rudolphi, 1819

Hosts: Tupinambis teguixin

Site of infection: Intestine

Distribution: Uruguay - MO $(29,71)$.

Cruzia travassosi Kalil \& Vogelsangi, 1932

Hosts: Tupinambis merianae

Site of infection: Intestine

Distribution: Brazil - Salobra (Pantanal) and Bodoquena (Cerrado) MS; Bolivia; Argentina (7, $41,71)$.
Falcaustra belemensis Baker \& Bain, 1981

Hosts: Neusticurus bicarinatus Linnaeus, 1758;

Potamites ecpleopus Cope, 1876; P. strangulatus Cope, 1868

Site of infection: Rectum

Distribution: Ecuador - PA (Amazon) (39).

Falcaustra sp.

Hosts: Anolis punctatus

Site of infection: Large intestine

Distribution: Brazil - AM (Amazon) (40).

\section{Seuratidae}

\section{Skrjabinelazia Sypliaxov, 1930}

Skrjabinelazia galliardi Chabaud, 1973

Hosts: Gonatodes humeralis Guichenot, 1855

Site of infection: Stomach

Distribution: Brazil - Belém (Amazon), PA (8, 74, 75).

Skrjabinellazia intermedia Freitas, 1940

Hosts: Anolis puncatus; Cnemidophorus nativo Rocha, Bergallo \& Peccinini-Seale, 1997; Tropidurus guarani; T. torquatus; Stenocercus caducus (Cope, 1862)

Site of infection: Stomach, intestine

Distribution: Brazil - Salobra (Pantanal) and Bodoquena (Cerrado), MS; Salvador and Guaratiba (Restinga), BA; Jurubatiba (Restinga), RJ; Paraguay - (Chaco); Peru - CU (Amazon). Comments: Originally described as Salobrella intermedia found in Tropidurus spinulosus from Salobra, MS. See comments on host taxonomy at Strongyluris oscari $(1,35,41,62,65,76,77)$.

\section{Subuluridae}

\section{Subulura Molin, 1860}

Subulura lacertilia Vicente, Van-Sluys, Fontes \& Kiefer, 2000

Hosts: Eurolophosaurus nanuzae, Cnemidophorus nativo

Site of infection: Intestine

Distribution: Brazil - Serra do Cipó (Cerrado), MG; Guaratiba (Restinga), BA $(66,77,78)$.

Subulura sp.

Hosts: Tropidurus torquatus

Site of infection: Small intestine

Distribution: Brazil - Jurubatiba (Restinga), RJ (35).

\section{Oxyurida}




\section{Oxyuridae}

Gynaecometra Araújo, 1976

Gynaecometra bahiensis Araújo, 1976

Hosts: Polychrus acutirostris Spix, 1825

Site of infection: Intestine

Distribution: Brazil - Xique-xique (Caatinga),

BA; Campo Grande (Cerrado), MS $(41,79)$.

\section{Typhlonema Kreis, 1938}

Typhlonema sp.

Hosts: Tropidurus guarani

Site of infection: Not informed

Distribution: Brazil - Salobra (Pantanal), MS (8).

\section{Pharyngodonidae}

\section{Alaeuris Thapar, 1925}

Alaeuris caudatus Lent \& Freitas, 1948

Hosts: Iguana iguana

Site of infection: Intestine

Distribution: Brazil - Exu (Caatinga), PE;

Venezuela - La Puerta, GU; Peru - TU $(8,29,80)$.

Alaeuris conolophi Cuckler, 1938

Hosts: Conolophus subcristatus Gray, 1831

Site of infection: Intestine

Distribution: Ecuador - Galapagos Islands (7).

Alaeuris galapagensis Cuckler, 1938

Hosts: Conolophus subcristatus Gray, 1831

Site of infection: Intestine

Distribution: Ecuador - Galapagos Islands (7).

Alaeuris labicula Cuckler, 1938

Hosts: Conolophus subcristatus

Site of infection: Intestine

Distribution: Ecuador - Galapagos Islands (7).

Alaeuris longispicula Cuckler, 1938

Hosts: Conolophus subcristatus

Site of infection: Intestine

Distribution: Ecuador - Galapagos Islands (7).

Alaeuris vogelsangi Lent \& Freitas, 1948

Hosts: Iguana iguana

Site of infection: Intestine

Distribution: Brazil - Exu (Caatinga), PE, CE;

Venezuela - La Puerta, GU $(8,29,81)$.

\section{Ozolaimus Dujardin, 1845}

Ozolaimus cirratus Linstow, 1906

Hosts: Iguana iguana

Site of infection: Intestine
Distribution: Brazil - Santa Luzia (Caatinga), PB;

Peru; Venezuela - La Puerta, GU, Blanquilla, Los Frailes, Los Testigos and Margarita; Colombia LG; Surinam - PR and Marienburg, CO (8, 29, $82,83)$.

Ozolaimus megatyphlon Rudolphi, 1819

Hosts: Iguana iguana

Site of infection: Intestine

Distribution: Brazil - Santa Luzia (Caatinga), PB;

Peru; Venezuela - La Puerta, GU, Blanquilla, Los

Frailes, Los Testigos and Margarita; Colombia -

LG; Surinam - PR and Marienburg, CO (8, 29, $82,83)$.

\section{Paralaeuris Cuckler, 1938}

Paralaeuris dorochila Cuckler, 1938

Hosts: Conolophus subcristatus

Site of infection: Intestine

Distribution: Ecuador - Galapagos Islands (7).

\section{Parapharyngodon Chatterji, 1933}

Parapharyngodon alvarengai Freitas, 1957

Hosts: Trachylepis atlantica, Ameiva ameiva, Amphisbaena ridleyi

Site of infection: Large intestine

Distribution: Brazil - Fernando de Noronha Island, PE and Itaguaí (Restinga), RJ.

Comments: after the synonym it was cited as Thelandros alvarengai $(46,84,85)$.

Parapharyngodon arequipensis Calisaya \& Córdova, 1997

Hosts: Microlophus peruvianus (Lesson, 1826)

Site of infection: Large intestine

Distribution: Peru - Omate, MO.

Comments: This species is apparently ignored by severeal authors (86-90).

Parapharyngodon largitor Alho \& Rodrigues, 1963

Hosts: Ameiva ameiva, Mabuya agilis, Hemidactylus mabouia, Dicrodon guttulatum

Site of infection: Intestine

Distribution: Brazil - Manguinhos and Grumari (Restinga), RJ and Valinhos (Cerrado), SP; PERU - LA $(8,22,33,91,92)$.

Parapharyngodon moqueguensis Calisaya \& Córdova, 1997

Hosts: Microlophus peruvianus, Dicrodon guttulatum 
Site of infection: Large intestine

Distribution: Peru - Moquegua, MO, LI, TU, PI, LA, LL, AN.

Comments: check $P$. arequipensis $(89,90,92)$.

Parapharyngodon riojensis Ramallo, Bursey \& Goldberg, 2002

Hosts: Phymaturus punae Cei, Etheridge \& Videla, 1985; P. palluma Molina, 1782; Liolaemus buergeri Werner, 1907

Site of infection: Large intestine

Distribution: Argentina - LR $(2,86)$.

Parapharyngodon sceleratus Travassos, 1923

Hosts: Ameiva ameiva; Cnemidophorus littoralis; Eurolophosaurus nanuzae; Hemidactylus mabouia; Kentropyx pelviceps; Liolaemus lutzae; Mabuya agilis; M. bistriata; M. caissara RebouçasSpieker, 1974; M. frenata; M. macrorhyncha; Microlophus albermalensis Baur, 1890; $P$. johnwrighti; Phyllopezus pollicaris; T. guarani; T. hispidus; T. itambere; T. semitaeniatus Spix, 1825; T. torquatus; T. melanopleurus and Tropidurus sp. Site of infection: Intestine

Distribution: Brazil - Abrolhos, Trancoso (Restinga) and Canudos, BA; CE; Linhares and Praia das Neves, ES; Serra do Cipó (Cerrado), MG; Salobra (Pantanal), MS; Xavantina, GO; Cachimbo, PA; Mogeiro, Lagoa Remígio, Umbuseiro and João Pessoa, PB; Garanhuns, PE; Grumari, Rio de Janeiro, Arraial do Cabo, Grussaí, Manguinhos and Maricá, RJ; Cruzeta, Currais Novos and Ceará Mirim (Caatinga), RN; Queimada Grande, Caraguatatuba and Valinhos, SP; Bolivia: Florida province, SC and El Carmen; Paraguay; Peru - CU; Ecuador - Galapagos Islands $(1,7,8,17,20,22,27,32-34,62,65,66$, 69, 81, 91, 93-97).

Comments: This species has been widely reported as $P$. scleratus $(1,86)$. However, according to the original description and the latter redescription, the proper spelling of the specific name is sceleratus $(59,93)$. Cnemidophorus occelifer, cited by Ribas et al. (94), actually corresponds to C. littoralis (31).

Parapharyngodon senisfaciecaudus Freitas, 1957 Hosts: Liolaemus signifer Duméril \& Bibron, 1837 Site of infection: Large intestine

Distribution: Bolivia - LP.

Comments: It was originally described infecting Liolaemus lenzi, which actually corresponds to $L$. signifier (98).
Parapharyngodon verrucosus Freitas \& Dobbin, 1959

Hosts: Diploglossus lessonae

Site of infection: Intestine

Distribution: Brazil - João Alfredo (Caatinga),

PE; CE (Caatinga) $(8,81,99)$.

Parapharyngodon yurensis Calisaya \& Córdova, 1997

Hosts: Microlophus peruvianus

Site of infection: Large intestine

Distribution: Peru - Yura, AR.

Comments: check $P$. arequipensis $(89,90)$.

\section{Parapharyngodon sp}

Hosts: Ameiva ameiva, Cnemidophorus nativo, Hemidactylus mabouia, Tropidurus torquatus, T. etheridgei, Dicrodon guttulatum

Site of infection: Intestine

Distribution: Brazil - Salobra, MS; Cabo Frio, Nova Iguaçu and Maricá, RJ, Salvador and Guaratiba, BA; Argentina - CO, SA; Peru - TU, LA, LI (8, 16, 21, 77, 92, 100-102).

\section{Pharyngodon Diesing, 1861}

Pharyngodon cesarpintoi Pereira, 1935

Hosts: Cnemidophorus sp., Ameiva ameiva, Liolaemus lutzae, Tropidurus itambere

Site of infection: Large intestine

Distribution: Brazil - Juazeiro and Mogeiro (Caatinga), PB, BA, RN; Maricá (Restinga), RJ; Bodoquena (Cerrado), MS.

Comments: The type of host is apparently misidentified in the original description (60). However, C. lemniscatus does not occur in the Caatinga biome, but $C$. occelifer and at least three undescribed species occur $(41,60,103,104)$.

Pharyngodon micrurus Freitas \& Ibañez, 1963

Hosts: Dicrodon heterolepis, D. guttulatum

Site of infection: Intestine

Distribution: Peru - Trujillo, LL; TU, PI, LA, AN, LI $(7,92,105)$.

Pharyngodon travassosi Pereira, 1935

Hosts: Ameiva ameiva

Site of infection: Large intestine

Distribution: Brazil - Areia (Caatinga), PB (60).

Pharyngodon sp.

Hosts: Tropidurus torquatus, Tropidurus hispidus Spix, 1825 
Site of infection: Intestine

Distribution: Brazil - Salvador, BA; CE $(8,81)$.

\section{Pseudostrongyluris Guerrero, 1971}

Pseudostrongyluris polychrus Guerrero, 1971

Hosts: Polychrus marmoratus

Site of infection: Not informed

Distribution: Venezuela - Federal District (7).

\section{Skrjabinodon Inglis, 1968}

Skrjabinodon dixoni Bursey \& Goldberg, 2007

Hosts: Uracentron flaviceps (Guichenot, 1855)

Site of infection: Large intestine

Distribution: Peru - LO (Amazon); Ecuador - SU (106).

Skrjabinodon heliocostai Vicente, Vrcibradic, Muniz-Pereira \& Pinto, 2000

Hosts: Mabuya frenata

Site of infection: Large intestine

Distribution: Brazil - Valinhos (Cerrado), SP (107).

Skrjabinodon spinulosus Vicente, Vrcibradic, Rocha \& Pinto, 2002

Hosts: Mabuya dorsivittata

Site of infection: Intestine

Distribution: Brazil - Itatiaia (Atlantic Forest), RJ; Itirapina (Cerrado), SP $(108,109)$.

Spauligodon Skrjabin, Schikhobalova \& Lagodovsk., 1960

Spauligodon loboi Ramallo, Bursey \& Goldberg (2002)

Hosts: Liolaemus capillitas Hulse, 1979; L. huacahuasicus Laurent, 1985; L. quilmes Etheridge, 1993; L. ornatus Koslowsky, 1898; L. ramirezae Lobo, 1999

Site of infection: Large intestine

Distribution: Argentina - Tafídel Valle and Amaicha del Valle, TU; El Cerrillo, Andalgalá, Belén, and Santa María, CA; San Antonio de los Cobres, Cachi, and La Poma, SA; and Tilcara and Humahuaca, JU (110).

Spauligodon maytacapaci Vicente \& Ibañez (1968) Hosts: Leiocephalus sp.; Liolaemus andinus Koslowsky, 1895; L. chilensis Lesson, 1830; L. elongatus Koslowsky, 1896; L. lemniscatus Gravenhorst, 1838; L. pictus Duméril \& Bibron, 1837; L. tenuis Duméril \& Bibron, 1837 Site of infection: Intestine
Distribution: Peru - Pumarongo, CA; Argentina; Chile $(2,111,112)$.

Spauligodon oxkutzcabiensis (Chitwood, 1938) Hosts: Thecadactylus solimoensis Bergmann \& Russell, 2007; Phyllodatylus reissii Peters, 1862; P. inaequalis Cope, 1876; P. johnwrighti; P. microphyllus Cope, 1876; Tropidurus guarani; Bogertia lutzae (Loveridge, 1941).

Site of infection: Intestine

Distribution: Peru - CU, (Amazon); Paraguay - Arroyo Corrientes (Chaco), PA; Brazil - AL (Atlantic Forest).

Comments: Thecadactylus was recently subject to revision, and T. solimoensis was described by Bergmann \& Russel in localities that encompasses the Cuzco, Peru (1, 65, 97, 113-115).

Spauligodon viracochai Freitas, Vicente \& Ibañez, 1968

Hosts: Phyllodactylus gerrhopygus Wiegmann, 1834; P. angustidigitus Dixon \& Huey, 1970

Site of infection: Intestine

Distribution: Peru - Trujilo, LL and IC $(116,117)$.

\section{Thelandros Wedl, 1862}

Thelandros bulbosus Salas \& Campos, 1974

Hosts: Microlophus peruvianus

Site of infection: Not informed

Distribution: Peru.

Comments: Despite the citation by Pérez et al. (117), this oxyurid was incorrect described in "Libro de Resumenes del IV Congreso Nacional de Biologia", a meeting abstract. Due to an inaccordance with articles 8.1 and 9.9 of the International Code for Zoological Nomenclature, it was therefore considered a species inquirenda $(107,118)$.

Thelandros capacyupanquii Freitas, Vicente \& Ibañez, 1968

Hosts: Dicrodon holmbergi Schmidt, 1957; Dicrodon guttulatum

Site of infection: Intestine

Distribution: Peru - Trujilo, LL, IC, AN (92, 107, 118).

Thelandros sp.

Hosts: Tropidurus etheridgei

Site of infection: Intestine

Distribution: Argentina - Departamento Anta (Chaco), SA (102). 


\section{Rhabditida}

\section{Rhabdiasidae}

Chabirenia Lhermitte Vallarino, Bain, Deharo, Bertani, Voza, Attout \& Gaucher, 2005

Chabirenia cayennensis Lhermitte Vallarino, Bain, Deharo, Bertani, Voza, Attout \& Gaucher, 2005

Hosts: Ameiva ameiva

Site of infection: Mouth

Distribution: French Guiana - CY (119).

\section{Rhabdias Stiles \& Hassall, 1905}

Rhabdias anolis Bursey, Goldberg \& Telford, 2003

Hosts: Anolis punctatus

Site of infection: Lungs

Distribution: Peru - CU (Amazon) (1).

Rhabdias elegans Gutierrez, 1945

Hosts: Anolis punctatus

Site of infection: Lungs

Distribution: Ecuador - PA (Amazon) (1).

\section{Rhabdias sp.}

Hosts: Anisolepis undulatus Wiegmann, 1834; Anolis fuscoauratus; A. punctatus; Enyalius iheringii; E. bilineatus; E. perditus; Tropidurus hispidus

Site of infection: Lungs, stomach

Distribution: Brazil - Marechal Floriano (Atlantic Forest), ES; PA, AM (Amazon); São Sebastião (Atlantic Forest), SP, Chapada do Araripe (Caatinga), CE; Ecuador - SU.

Comments: Of the neotropical lizards, only Rhabdias anolis has been described, despite several reports in the same region $(3,24,40,51$, $120,121)$.

\section{Strongyloididae}

\section{Strongyloides Grassi, 1897}

Strongyloides cruzi Rodrigues, 1968

Hosts: Hemidactylus mabouia

Site of infection: Small intestine

Distribution: Brazil - Manguinhos, RJ $(8,122)$.

\section{Spirurida}

\section{Acuariidae}

Hosts: Cercosaura argulus; Hemidactylus mabouya; Mabuya agilis; M. macrorhyncha; Tropidurus torquatus; Enyalius bilineatus; Phyllodactylus lepidopygus Tschudi, 1845

Site of infection: Body cavity

Distribution: Brazil - Queimada Grande Island (Atlantic Forest) and Valinhos (Cerrado), SP;
Jurubatiba and Grussaí (Restinga), RJ; Marechal Floriano (Atlantic Forest) and Praia das Neves (Restinga), ES; Peru - Rio Nanay, LO.

Comments: all aforementioned citations consist of cysts and thus difficult the identification to species level since reproductive structures are required $(20,22-24,35,45)$.

\section{Diplotriaenidae \\ Hastospiculum Skrjabin, 1923 \\ Hastospiculum sp. \\ Hosts: Plica umbra \\ Site of infection: Peritoneum \\ Distribution: Peru - CU (Amazon) (1).}

\section{Filariidae}

\section{Filaria Mueller, 1787}

Filaria multipapilla Molin,

Hosts: Dracaena guianensis Daudin, 1802

Site of infection: Body cavity

Distribution: Brazil - Belém (Amazon), PA.

Comments: The species is actually considered species inquirenda, because these first records were lost and no new information about the species has been presented since the description (123-125).

\section{Onchocercidae}

\section{Macdonaldius Khanna, 1933}

Macdonaldius grassi (Caballero, 1954)

Hosts: Phyllopezus pollicaris (Spix, 1825)

Site of infection: Underneath the epidermis of the throat

Distribution: Paraguay - CO (Chaco) (126).

\section{Oswaldofilaria Travassos, 1933}

Oswaldofilaria azevedoi Bain, 1974

Hosts: Polychrus marmoratus Linnaeus, 1758; Stenocercus roseiventris

Site of infection: Body cavity

Distribution: Brazil - Belém (Amazon), PA; Peru - CU (Amazon) (1, 127).

Oswaldofilaria belemensis Bain and Dulahian, 1974

Hosts: Dracaena guianensis

Site of infection: Heart, aorta e vena cava

Distribution: Brazil - Belém (Amazon), PA (8).

Oswaldofilaria brevicaudata Rodhain and Vuylsteke, 1937

Hosts: Iguana iguana, Anolis punctatus 
Site of infection: Body cavity

Distribution: Brazil - Marajó (Amazon), PA, AM

(Amazon), Exu (Caatinga), PE; Venezuela - ZU

$(7,40,125,127-129)$.

Oswaldofilaria petersi Bain and Sulahian, 1974

Hosts: Tupinambis teguixin, Tropidurus hispidus

Site of infection: Body cavity

Distribution: Brazil - Belém (Amazon), PA,

Ibiraba (Caatinga), BA $(8,130)$.

Oswaldofilaria spinosa Bain and Sulahian, 1974

Hosts: Mabuya bistriata

Site of infection: Body cavity

Distribution: Brazil - Belém (Amazon) (8).

Oswaldofilaria sp.

Hosts: Tupinambis teguixin, Mabuya frenata

Site of infection: Body cavity

Distribution: Surinam - PR; Brazil - Valinhos

(Cerrado), SP $(28,34)$.

\section{Piratuba Freitas \& Lent, 1947}

Piratuba digiticauda Lent \& Freitas, 1941

Hosts: Tropidurus torquatus, Tropidurus guarani, Plica umbra, Kentropyx calcarata

Site of infection: Body cavity, intestine

Distribution: Brazil - Salvador, BA; Piratuba (Amazon), PA; Salobra (Pantanal), MS; Juara (Amazon), MT; Peru - CU; Paraguay - Chaco.

Comments: Tropidurus spinulosus found in Salobra actually correspond to T. guarani $(1,62$, $63,65,125,131)$.

\section{Piratuba lainsoni Bain, 1974}

Hosts: Anolis punctatus, Polychrus marmoratus

Site of infection: Body cavity, large intestine Distribution: Brazil - Belém (Amazon), PA; Peru

- CU (Amazon) (1, 127).

Piratuba scaffi Bain, 1974

Hosts: Ameiva ameiva

Site of infection: Body cavity

Distribution: Brazil - Belém (Amazon), PA (127).

Piratuba shawi Bain, 1974

Hosts: Kentropyx calcarata Spix, 1825

Site of infection: Body cavity

Distribution: Brazil - Belém (Amazon), PA (127).

\section{Piratuboides Bain \& Sulahian, 1974}

Piratuboides zeae (Bain, 1974) Bain and Sulahian,

\section{4}

Hosts: Mabuya bistriata Spix, 1825

Site of infection: Body cavity, large intestine

Distribution: Brazil - Belém (Amazon), PA; Peru

- CU (Amazon) (1, 127).

\section{Physalopteridae}

\section{Abbreviata Travassos, 1920}

Abbreviata spiralis (Schneider, 1866) Chabaud, 1956

Hosts: Amphisbaena sp.

Site of infection: Not informed

Distribution: Brazil

Comments: This species is considered a species inquirenda (7).

\section{Physaloptera Rudolphi, 1819}

Physaloptera lutzi Cristofaro, Guimarães \& Rodrigues, 1976

Hosts: Ameiva ameiva; Cnemidophorus abaetensis Reis, Dias, Rocha \& Vrcibradic, 2002; C. littoralis; Enyalius bilineatus; Eurolophosaurus nanuzae; Liolaemus alticolor Barbour 1909; L. ornatus; L. quilmes; Tropidurus guarani; T. itambere; T. torquatus

Site of infection: Stomach

Distribution: Argentina - SA, TU; Bolivia Roboré (Pantanal); Brazil - Salvador, Serrinha and Canudos (Caatinga), BA; Linhares and Conceição da Barra (Restinga), Marechal Floriano (Atlantic Forest), ES; Serra do Cipó (Cerrado), MG; Porto Esperança and Salobra (Pantanal), MS; Xavantina, MT; Cachimbo (Amazon), PA; Maricá and Jurubatiba (Restinga), RJ; Valinhos (Cerrado), SP; Paraguay - Chaco (8, 24, 31, 32, $35,62,65,66,94-96,132,133)$.

Comments: Cnemidophorus occelifer, cited by Ribas et al. (94), actually corresponds to $C$. littoralis (31).

Physaloptera retusa Rudolphi, 1819

Hosts: Ameiva ameiva; Ameiva festiva Lichtenstein, 1856; Amphisbaena alba; Anolis fuscoauratus; Anolis punctatus; Cnemidophorus abaetensis; C. lemniscatus; C. littoralis; C. native; C. ocellifer; Enyalius bilineatus; Iguana iguana; Kentropyx altamazonica; K. calcarata; K. pelviceps; Leiosaurus bellii Duméril \& Bibron, 1837; L. catamarcensis Koslowsky, 1898; Liolaemus lutzae; L. neuquensis Cei \& Videla, 2003; Mabuya agilis; M. bistriata; M. dorsivittata Cope, 1862; Ophiodes striatus Spix, 1824; Plica plica; P. umbra; Potamites 
ecpleopus; Stenocercus roseiventris; Thecadacytlus solimoensis; Tropidurus guarani; T. hispidus; T. torquatus; Tupinambis rufescens Günther, 1871; T. longilineus Ávila-Pires, 1995; T. teguixin; Uracentron flaviceps; Tropidurus melanopleurus Boulenger, 1902

Site of infection: Stomach, intestine

Distribution: Brazil - Salvador and Guaratiba (Restinga), BA; Conceição da Barra (Restinga) and Marechal Floraino (Atlantic Forest), ES; Salobra (Pantanal), MS; Juara (Amazon), MT; Altamira, Belém, Cachimbo, Novo Progresso and Santarém (Amazon), PA; Itatiaia (Atlantic Forest), Maricá and Jurubatiba (Restinga), RJ; Ilha Seca (Atlantic Forest) and Itirapina (Cerrado), SP; Paraguay - Chaco; Bolivia - El Carmen and Florida, SC; Argentina; Peru - CU (Amazon); Surinam - PR (Amazon); Uruguay - Montevideo; Venezuela - Colón, ZU; Atures, AM; Salamanca, NE; Cumuná (Arid zone) and La Orchila, Mar Caribe; Colombia - $\mathrm{CH}$.

Comments: Baker(7) cited severalspecies oflizards that does not occur in Brazil, such as Tupinambis rufescens, Cnemidophorus lateristrigus, Euprepis spixii, Podinema graphica and P. scripta. According to the same author, Pygopus gronovii actually corresponds to Ophiodes striatus and Podinema corresponds to Tupinambis, but no species are currently recognized as synonyms of $P$. scripta and P. graphica (7). Cnemidophorus occelifer, cited by Ribas et al. (94), actually corresponds to C. littoralis $(1,8,24,28,29,31,32,35,51,57,62$, $69,77,94,108,126,131,134-142)$.

Physaloptera sp.

Hosts: Ameiva ameiva; Cercosaura argulus; Cnemidophorus litoralis; C. ocellifer; Hemidactylus mabouia; Hoplocercus spinosus; Mabuya agilis; $M$. macrorhyncha; Polychrus acutirostris; Tropidurus etheridgei; T. torquatus; Tupinambis merianae Duméril \& Bibron, 1839; Tupinambis teguixin; Dicrodon guttulatum.

Site of infection: Stomach

Distribution: Brazil - Abrolhos and Salvador (Restinga), BA; Linhares and Praia das Neves (Restinga) and Sooretama (Cerrado), ES; Salobra (Pantanal) and Bodoquena (Cerrado), MS; Ilha Grande, Cabo Frio, Grussaí, Grumari and Jurubatiba (Restinga), RJ; Valinhos (Cerrado), SP; Alegria (Pampas), RS; Argentina - SA and FO (Chaco); Peru - Rio Nanay, LO, LI.

Comments: Tupinambis teguixin of Salobra,
MS corresponds to $T$. merianae (personal observation) $(8,16,22,31,33,35,41,45,92,96$, $101,102,143)$.

\section{Physalopteroides Wu \& Liu, 1940}

Physalopteroides venancioi Lent, Freitas \& Proença, 1946

Hosts: Alopoglossus atriventris Duellman, 1973; Ameiva ameiva; Cercosaura ocellata Wagler, 1830; Cnemidophorus native; Kentropyx altamazonica; K. calcarata; K. pelviceps; Mabuya agilis; $M$. bistriata; M. macrorhyncha; Thecadactylus solimoensis; Tropidurus torquatus; Tupinambis teguixin

Site of infection: Stomach

Distribution: Brazil - AC (Amazon); Trancoso and Guaratiba (Restinga), BA; Juara (Amazon), MT; Magé, Grussaí and Jurubatiba (Restinga), RJ; Praia das Neves (Restinga), ES; Peru - CU (Amazon) $(1,17,23,33,35,52,77,131,144)$.

\section{Thubunaea Seurat, 1914}

Thubunaea iguanae (Telford, 1965)

Hosts: Microlophus peruvianus; M. thoracicus (Tschudi, 1845)

Site of infection: Stomach

Distribution: Peru - R. N. Paracas, IC (117).

Thubunaea parkeri Baylis, 1926

Hosts: Microlophus occipitalis Peters, 1871;

Dicrodon heterolepis

Site of infection: Stomach

Distribution: Peru $(7,145)$.

\section{Spiruridae}

Spirurinae

Hosts: Hemidactylus mabouia

Site of infection: Stomach, lungs, and small intestine

Distribution: Brazil - Rio de Janeiro, RJ (146).

\section{Micropleuridae}

Micropleura Linstow, 1906

Hosts: Tupinambis meriana

Site of infection: Body cavity

Distribution: Brazil - Salobra (Pantanal), MS.

Comments: This record must be discharged, because no mention of Micropleura was found in Travassos and Freitas (147) as cited by Vicente et al. (8). Moreover, Micropleura may be found in the body cavity of crocodiles and turtles (148). 
Diaphanocephalidae

Diaphanocephalus Diesing, 1851

Diaphanocephalus diesingi Freitas \& Lent, 1938

Hosts: Tupinambis teguixin

Site of infection: Small intestine

Distribution: Brazil - Belém (Amazon), PA (149).

Diaphanocephalus galeatus Rudolphi, 1819

Hosts: Tupinambis merianae, T. rufescens, $T$. teguixin

Site of infection: Intestine

Distribution: Brazil - Salobra (Pantanal) and Bodoquena (Cerrado), MS; Manguinhos, Angra dos Reis e Rio de Janeiro (Atlantic Forest), RJ; Pedras Altas, RS; Ilha Grande and São Paulo, SP, Fernando de Noronha, PE; Argentina; Bolivia; Surinam - PR $(8,28,46,149,150)$.

Diaphanocephalus jacuruxi Alho, 1965

Hosts: Dracaena guianensis

Site of infection: Small intestine

Distribution: Brazil - Ilha das Onças (Amazon), PA $(8,151)$.

\section{Kalicephalus Molin, 1861}

Kalicephalus sp.

Hosts: Tupinambis teguixin

Site of infection: Intestine

Distribution: Brazil - Sooretama, ES; Alegria (Pampas), RS.

Comments: Species of Kalicephalus spp. have been reported in several snake species, and seems to be restricted to these animals, likewise Diaphanocephalus spp. (a sister taxon) in Tupinambinae. Published records could be a misidentification or a case of incidental infection in a Tupinambis teguixin $(8,143,152)$.

\section{Molineidae}

\section{Kentropyxia Baker, 1982}

Kentropyxia sauria Baker, 1982

Hosts: Kentropyx calcarata

Site of infection: Small intestine

Distribution: Brazil - Belém and Novo Progresso

(Amazon), PA (136, 153).

\section{Oswaldocruzia Travassos, 1917}

Oswaldocruzia bainae Ben-Slimane \& Durette-

Desset, 1996

Hosts: Anolis chrysolepis Duméril \& Bibron, 1837;

A. fuscoauratus

Site of infection: Small intestine
Distribution: Ecuador - San Pablo (Amazon), LR (154).

Oswaldocruzia benslimanei Durette-Desset, Anjos \& Vrcibradic, 2006

Hosts: Enyalius bilineatus Duméril \& Bibron, 1837

Site of infection: Small intestine

Distribution: Brazil - Marechal Floriano, ES (24, 155).

Oswaldocruzia brasiliensis Lent \& Freitas, 1935

Hosts: Hemidactylus mabouia

Site of infection: Small intestine

Distribution: Brasil - Rio de Janeiro, RJ $(8,156)$.

Oswaldocruzia burseyi Durette-Desset, Anjos \& Vrcibradic, 2006

Hosts: Enyalius perditus

Site of infection: Stomach

Distribution: Brazil - São Sebastião (Atlantic

Forest), SP $(3,155)$.

Oswaldocruzia fredi Durette-Desset, Anjos \& Vrcibradic, 2006

Hosts: Enyalius iheringii

Site of infection: Stomach

Distribution: Brazil - São Sebastião (Atlantic

Forest), SP $(3,155)$.

Oswaldocruzia peruensis Ben-Slimane, Verhaag \& Durette-Desset, 1995

Hosts: Stenocercus roseiventris, Cercosaura argulus, Anolis punctatus

Site of infection: Stomach

Distribution: Peru - HA and CU (Amazon) (1, $45,157)$.

Oswaldocruzia vitti Bursey \& Goldberg, 2004

Hosts: Alopoglossus angulatus, A. atriventris, Anolis fuscoauratus, A. punctatus, Cercosaura eigenmanni, C. oshaugnessyi

Site of infection: Intestine

Distribution: Brazil - AC, AM, PA, RO (Amazon); Ecuador - SU (Amazon); Peru - CU (Amazon) $(1,18,40,51,52)$.

Oswaldocruzia sp.

Hosts: Tropidurus torquatus, Enyalius catenatus (Wied, 1821), E. perditus, K. calcarata

Site of infection: Intestine

Distribution: Brazil - Bodoquena (Cerrado) 
and Salobra (Pantanal), MS; Juara (Amazon), MT; Cachimbo (Amazon), PA; Rio de Janeiro, RJ; Ibitipoca, MG; Argentina - SA, JU and TA (Chaco); Paraguay - Assuncion and Chaco.

Comments: The aforementioned records of T. torquatus are those of O. mazzai and that of Enyalius spp. are from O. subauricularis, both considered species inquirenda $(48,131,155,158)$.

\section{Cestoda}

Cyclophyllidea

Linstowiidae

\section{Oochoristica Lühe, 1898}

Oochoristica ameivae (Beddard, 1914)

Hosts: Ameiva ameiva, Cnemidophorus nativo, Mabuya agilis, M. macrorhyncha

Site of infection: Intestine

Distribution: Brazil - Cachimbo (Amazon), PA; Serra do Navio (Amazon), AP; Manaus (Amazon), AM; Guaratiba and Trancoso (Restinga), BA; Praia das Neves (Restinga), ES; Grussaí (Restinga), RJ; Peru - CU (Amazon) (1, $17,23,77,159-161)$.

Oochoristica bressalui Fürhmann, 1927

Hosts: Tropidurus guarani, T. hispidus, T. torquatus Site of infection: Small intestine

Distribution: Brazil - Salvador and Serrinha, BA; Cachimbo (Amazon), Arraial do Cabo, Cabo Frio and Rio de Janeiro, RJ; Bolivia - El Carmen; Paraguay $(19,65,160,162)$.

Oochoristica freitasi Rego \& Ibañez, 1965

Hosts: Dicrodon heterolepis; D. guttulatum Duméril \& Bibron, 1893

Site of infection: Small intestine

Distribution: Peru - Trujillo, LL; TU, PI.

Comments: Bursey and Goldberg (163) and Arizmendi-Espinosa et al. (164) apparently ignored the presence of $O$. freitasi in their findings of Oochoristica spp. infecting lizards in neotropical regions. The species is not included in any synonymy of the revisions made by Bursey et al. (165). Moreover, the species was cited in Guillén-Hernández et al. (166).

Oochoristica iguanae (Baylis, 1919) Bursey \& Goldberg, 1996

Hosts: Iguana iguana, Dicrodon guttulatum

Site of infection: Small intestine

Distribution: Venezuela - Isla Margarita; Peru LA, PI $(92,163)$.
Oochoristica travassosi Rego \& Ibañez, 1965

Hosts: Leiocephalus sp.; Liolaemus vallecurensis

Pereira, 1992; Phyllodactylus johnwrigthi Dixon \& Huey, 1970

Site of infection: Small intestine

Distribution: Peru - Moche; Argentina - SJ (2, 97, 167).

Oochoristica insulamargaritae López-Neyra and Diaz-Ungría, 1957

Hosts: Ameiva ameiva

Site of infection: Small intestine

Distribution: Venezuela - Isla Margarita (163, 168).

Oochoristica vanzolinii Rego \& Rodrigues, 1965

Hosts: Hemidactylus mabouia, Eurolophosaurus nanuzae

Site of infection: Small intestine

Distribution: Brazil - Rio de Janeiro, RJ; Serra do

Cipó, MG $(66,162)$.

Oochoristica sp.

Hosts: Alopoglossus atriventris, Amphisbaena ridleyi, Mabuya frenata, Tupinambis merianae, Trachylepis atlantica

Site of infection: Small intestine

Distribution: Ecuador - SU; Brazil - Valinhos, SP; Fernando de Noronha Islands, PE (34, 46, 52).

\section{Semenoviella Spasskii, 1951}

Semenoviella amphisbaenae Rudolphi, 1819

Hosts: Amphisbaena fuliginosa Linnaeus, 1758; A. alba

Site of infection: Intestine

Distribution: Brazil - Belém (Amazon), PA; Pirassununga (Cerrado), SP (169).

\section{Mesocestoididae}

\section{Mesocestoides Vaillant, 1863}

Mesocestoides sp.

Hosts: Anolis transversalis

Site of infection: Not informed

Distribution: Brazil - RO (Amazon) (40).

\section{Proteocephalidea}

Proteocephalidae

Cairaella Coquille \& De Chambrier, 2008

Cairaella henrii Coquille \& De Chambrier, 2008

Hosts: Anolis trachyderma Cope, 1876

Site of infection: Intestine 
Distribution: Ecuador - San Pablo de Kantesyia, SU (170).

\section{Ophiotaenia La Rue, 1911}

Ophiotaenia flava Rudin, 1917

Hosts: Kentropyx pelviceps

Site of infection: Not informed

Distribution: Peru - CU (Amazon) (1).

Ophiotaenia nicoleae Coquille \& De Chambrier, 2008

Hosts: Thecadactylus solimoensis

Site of infection: Intestine

Distribution: Ecuador - San Pablo de Kantesyia, SU.

Comments: Thecadactylus was recently subjected to revision, and a T. solimoensis was found in from localities that encompass San Pablo de Kantesyia, Ecuador $(113,170)$.

Ophiotaenia sp.

Hosts: Anolis fuscoauratus

Site of infection: Small intestine

Distribution: Brazil - Santarém (Amazon), PA (51).

\section{Tejidotaenia Freze, 1965}

Tejidotaenia appendiculata (Baylis, 1947)

Hosts: Tupinambis teguixin

Site of infection: Small intestine

Distribution: Surinam - PR (Amazon); Brazil -

Serra do Navio (Amazon), AP; Linhares, ES (28, 171).

\section{Trematoda}

Digenea

Echinostomata

Echinostomida

Superfamily Echinostomatoidea Looss, 1899

\section{Cathaemasiidae}

\section{Pulchrossomoides Freitas \& Lent, 1937}

Pulchrosomoides elegans Freitas \& Lent, 1937

Hosts: Tupinambis teguixin, Iguana iguana, Mabuya macrorhyncha

Site of infection: Stomach

Distribution: Brazil - Porto Esperança (Pantanal), MS; Praia das Neves (Restinga), ES (6, 23, 172, 173).

\section{Plagiorchiida}

Dicrocoeliidae
Brachycoelium (Dujardin, 1845)

Brachycoelium salamandrae (Frolich, 1789)

Dujardin, 1845

Hosts: Leposoma osvaldoi Ávila-Pires, 1995; Anolis nitens Wagler, 1830

Site of infection: Small intestine

Distribution: Brazil - Novo Progresso (Amazon), PA.

Comments: Cited as B. mesocoeliiformis when found infecting Anolis scypheus (A. nitens) (6, 137).

\section{Platynosomum}

Platynosomum sp. Looss, 1906

Hosts: Amphisbaena ridleyi, Trachylepis atlantica

Site of infection: Gallbladder, bile duct

Distribution: Brazil - Fernando de Noronha Island, PE (46).

\section{Harmotrematidae}

Helicotrema Odhner, 1912

Helicotrema asymetricum (Travassos, 1922)

Viana, 1924

Hosts: Iguana iguana

Site of infection: Intestine

Distribution: Brazil - Pantanal (174, 175).

Helicotrema magniovatum Odhner, 1912

Hosts: Iguana iguana

Site of infection: Intestine

Distribution: Brazil (6).

Helicotrema spirale (Diesing, 1850) Odhner, 1912

Hosts: Iguana iguana

Site of infection: Intestine

Distribution: Brazil (6).

\section{Pronocephalidae}

Iguanacola Gilbert, 1938

Iguanacola navicularis Gilbert, 1938

Hosts: Amblyrhynchus cristatus Bell, 1825

Site of infection: Not informed

Distribution: Ecuador - Galapagos Islands (14).

\section{Myosaccus Gilbert, 1938}

Myosaccus amblyrhynchi Gilbert, 1938

Hosts: Amblyrhynchus cristatus

Site of infection: Not informed

Distribution: Ecuador - Galapagos Islands (14).

Cetiosaccus Gilbert, 1938

Cetiosaccus galapagensis Gilbert, 1938 
Hosts: Amblyrhynchus cristatus

Site of infection: Not informed

Distribution: Ecuador - Galapagos Islands (14).

\section{Paradistomum Kossack, 1910}

Paradistomum parvissimum (Travassos, 1918)

Hosts: Ameiva ameiva, Hemidactylus mabouia, Iguana iguana, Liolaemus lutzae, Mabuya macrorhyncha, M. agilis, Tropidurus torquatus, Tupinambis teguixin

Site of infection: Gallbladder, liver, small intestine Distribution: Brazil - Arraial do Cabo, Grussaí, Nova Iguaçu, Manguinhos, Maricá, RJ; Praia das Neves (Restinga) and Santa Teresa, ES; Salvador and Trancoso (Restinga), BA (17, 19, 21, 23, 27, $100,146,176,177)$.

Paradistomum rabusculum Kossack, 1910

Hosts and records: Gymnodactylus geckoides Spix, 1825

Site of infection: Not informed

Distribution: Brazil (6).

\section{Macroderidae}

\section{Pneumotrema Bhalerao, 1937}

Pneumotrema travassosi Bhalerao, 1937

Hosts and records: Amphisbaena alba

Site of infection: Kidney, ureter, intestine.

Distribution: Brazil (172).

\section{Family Plagiorchiidae}

\section{Allopharynx (Strom, 1928)}

Allopharynx daileyi Bursey, Goldberg \& Vitt, 2005

Hosts: Uranoscodon superciliosus

Site of infection: Small intestine

Distribution: Brazil - PA, RO, RR (Amazon) (50).

\section{Dasymetra Nicoll, 1911}

Dasymetra tupinambis Nasir \& Diaz, 1971

Hosts: Tupinambis teguixin

Site of infection: Intestine

Distribution: Venezuela - Sucre (178).

\section{Styphlodora Looss, 1899}

Styphlodora condita Faria, 1911

Hosts: Tupinambis rufescens (Günther, 1871)

Site of infection: Kidney, ureter, intestine

Distribution: Argentina (9).

Plagiorchis Lühe, 1899

Plagiorchis freitasi Vicente, 1978

Hosts: Tropidurus torquatus
Site of infection: Small intestine

Distribution: Brazil - Conceição da Barra, ES (19).

Plagiorchis vicentei Rodrigues, 1994

Hosts: Hemidactylus mabouia

Site of infection: Small intestine

Distribution: Brazil - Teresópolis, RJ (179).

Family Brachycoeliidae

Mesocoelium Odhner, 1910

Mesocoelium monas (Rudolphi, 1819) Freitas, 1958

Hosts: Alopoglossus angulatus, Amphisbaena sp., Amphisbaena ridleyi, Cercosaura eigenmanni, Diploglossus lessonae, Trachylepis atlantica, Leposternon microcephalum, Tropidurus torquatus, Uranoscodon superciliosus

Site of infection: Intestine

Distribution: Brazil - PA, RO, RR (Amazon); João Alfredo (Caatinga) and Fernando de Noronha, PE; Maricá and Rio de Janeiro (Restinga), RJ; Ecuador - SU (Amazon) (6, 18, 46, 50, 52, 100).

Family Urotrematidae

Urotrema Braun, 1900

Urotrema shirleyae Zamparo, Brooks \& Tkach, 2005

Hosts: Anolis fuscoauratus

Site of infection: Small intestine

Distribution: Brazil - Santarém (Amazon), PA (51).

\section{HOST-PARASITE LIST}

Family Amphisbaenidae

Amphisbaena alba

Maracaya belemensis

Maracaya graciai

Aplectana albae

Aplectana raillieti

Physaloptera retusa

Semenoviella amphisbaenae

Pneumotrema travassosi

\section{Amphisbaena bolivica}

Aplectana tucumanensis

\section{Amphisbaena fuliginosa}

Semenoviella amphisbaenae

Amphisbaena ridleyi 
Aplectana albae

Parapharyngodon alvarengai

Mesocoelium monas

Platynosomum sp.

Oochoristica sp.

\section{Amphisbaena sp.}

Maracaya pusilla

Mesocoelium monas

\section{Leposternon microcephalum}

Cosmocerca rara

Mesocoelium monas

Paradollfusnema amphisbaenia

Family Iguanidae

Amblyrhynchus cristatus

Iguanacola navicularis

Myosaccus amblyrhynchi

Cetiosaccus galapagensis

\section{Iguana iguana}

Alaeuris caudatus

Alaeuris vogelsangi

Ozolaimus cirratus

Ozolaimus megatyphlon

Oswaldofilaria brevicaudata

Physaloptera retusa

Oochoristica iguanae

Pulchrosomoides elegans

Paradistomum parvissimun

Helicotrema asymetricum

Helicotrema magniovatum

Helicotrema spirale

\section{Conolophus subcristatus}

Alaeuris conolophi

Alaeuris galapagensis

Alaeuris labicula

Alaeuris longispicula

Paralaeuris dorochila

Family Hoplocercidae

Enyalioides praestabilis

Spinicauda spinicauda

\section{Hoplocercus spinosus}

Africana dardanelosi

Cruzia rudolphii

Physaloptera sp.

\section{Morunasaurus annularis}

Raillietnema brachyspiculatum

Family Polychrotidae

Anisolepis undulatus

Rhabdias sp.

Anolis chrysolepis

Oswaldocruzia bainae

Anolis fuscoauratus

Cosmocerca vrcibradici

Strongyluris oscari

Rhabdias sp.

Physaloptera retusa

Oswaldocruzia bainae

Oswaldocruzia vitti

Ophiotaenia sp.

Urotrema shirleyae

Anolis nitens

Brachycoelium salamandrae

Anolis punctatus

Cyrtosomum longicaudatum

Strongyluris oscari

Falcaustra sp.

Skrjabinellazia intermedia

Rhabdias anolis

Rhabdias elegans

Rhabdias sp.

Oswaldofilaria brevicaudata

Piratuba lainsoni

Physaloptera retusa

Oswaldocruzia peruensis

Oswaldocruzia vitti

Anolis trachyderma

Cairaella henrii

Anolis transversalis

Cyrtosomum longicaudatum

Strongyluris oscari

Mesocestoides sp.

Polychrus acutirostris

Gynaecometra bahiensis

Physaloptera sp.

Polychrus marmoratus

Pseudostrongyluris polychrus

Oswaldofilaria azevedoi

Piratuba lainsoni 
Family Leiosauridae

Enyalius bilineatus

Centrorhynchidae

Rhabdias sp.

Acuariidae

Physaloptera lutzi

Physaloptera retusa

Oswaldocruzia benslimanei

Enyalius catenatus

Oswaldocruzia sp.

Enyalius iheringii

Strongyluris oscari

Rhabdias sp.

Oswaldocruzia fredi

Enyalius perditus

Acanthocephalus sp.

Aplectana vellardi

Cosmocerca sp.

Strongyluris oscari

Rhabdias sp.

Oswaldocruzia burseyi

Oswaldocruzia sp.

Leiosaurus belli

Physaloptera retusa

Leiosaurus catamarcensis

Physaloptera retusa

Family Liolaemidae

Liolaemus alticolor

Physaloptera lutzi

\section{Liolaemus andinus}

Spauligodon maytacapaci

Liolaemus buergeri

Parapharyngodon riojensis

\section{Liolaemus capillitas}

Spauligodon loboi

Liolaemus chilensis

Spauligodon maytacapaci

Liolaemus elongatus

Spauligodon maytacapaci

Liolaemus huacahuasicus
Spauligodon loboi

\section{Liolaemus lemniscatus}

Spauligodon maytacapaci

\section{Liolaemus lutzae}

Ichthyocapillaria (= Pseudocapillaria) maricaensis

Parapharyngodon sceleratus

Pharyngodon cesarpintoi

Physaloptera retusa

Paradistomum parvissimum

\section{Liolaemus neuquensis}

Physaloptera retusa

Liolaemus ornatus

Spauligodon loboi

Physaloptera lutzi

Liolaemus pictus

Spauligodon maytacapaci

Liolaemus quilmes

Spauligodon loboi

Physaloptera lutzi

Liolaemus ramirezae

Spauligodon loboi

Liolaemus signifer

Parapharyngodon senisfaciecaudus

\section{Liolaemus tenuis}

Spauligodon maytacapaci

Liolaemus vallecurensis

Oochoristica travassosi

Phymaturus palluma

Parapharyngodon riojensis

Phymaturus punae

Parapharyngodon riojensis

\section{Family Tropiduridae}

Eurolophosaurus nanuzae

Strongyluris oscari

Subulura lacertília

Parapharyngodon sceleratus

Physaloptera lutzi

Oochoristica vanzolinii 


\section{Leiocephalus sp.}

Spauligodon maytacapaci

Oochoristica travassosi

Microlophus albermalensis

Parapharyngodon sceleratus

Microlophus occipitalis

Thubunaea parkeri

Microlophus peruvianus

Parapharyngodon arequipensis

Parapharyngodon moqueguensis

Parapharyngodon yurensis

Thubunaea iguanae

\section{Microlophus thoracicus}

Thubunaea iguanae

\section{Plica plica}

Strongyluris oscari

Physaloptera retusa

\section{Plica umbra}

Strongyluris oscari

Hastospiculum sp.

Piratuba digiticauda

Physaloptera retusa

\section{Stenocercus caducus}

Skrjabinellazia intermedia

Strongyluris oscari

\section{Stenocercus roseiventris}

Atractis cruciata

Strongyluris oscari

Oswaldofilaria azevedoi

Physaloptera retusa

Oswaldocruzia peruensis

\section{Tropidurus etheridgei}

Parapharyngodon sp.

Thelandros sp.

Physaloptera sp.

\section{Tropidurus guarani}

Strongyluris oscari

Skrjabinellazia intermedia

Typhlonema sp.

Parapharyngodon sceleratus

Spauligodon oxkutzcabiensis

Piratuba digiticauda
Physaloptera lutzi

Physaloptera retusa

Oochoristica bressalui

\section{Tropidurus hispidus}

Parapharyngodon sceleratus

Pharyngodon sp.

Rhabdias sp.

Oswaldofilaria petersi

Physaloptera retusa

Oochoristica bressalui

\section{Tropidurus itambere}

Parapharyngodon sceleratus

Pharyngodon cesarpintoi

Physaloptera lutzi

\section{Tropidurus melanopleurus}

Strongyluris oscari

Parapharyngodon sceleratus

Physaloptera retusa

\section{Tropidurus semitaeniatus}

Parapharyngodon sceleratus

\section{Tropidurus spinulosus}

Strongyluris oscari

\section{Tropidurus torquatus}

Acanthocephala

Echinorhynchus sp.

Centrorhynchus tumidulus

Capillaria (= Amphibiocapillaria) freitaslenti

Hexametra boddaertii

Strongyluris oscari

Skrjabinellazia intermedia

Subulura sp.

Parapharyngodon sceleratus

Parapharyngodon sp.

Pharyngodon sp.

Acuariidae

Piratuba digiticauda

Physaloptera lutzi

Physaloptera retusa

Physaloptera sp.

Physalopteroides venancioi

Oswaldocruzia sp.

Oochoristica bressalui

Paradistomum parvissimum

Plagiorchis freitasi

Mesocoelium monas 


\section{Tropidurus sp.}

Strongyluris oscari

Parapharyngodon sceleratus

Uracentron flaviceps

Skrjabinodon dixoni

Physaloptera retusa

Uranoscodon superciliosus

Cosmocerca vrcibradici

Africana chabaudi

Allopharynx daileyi

Mesocoelium monas

Family Gekkonidae

Hemidactylus mabouia

Centrorhynchidae

Parapharyngodon largitor

Parapharyngodon sceleratus

Parapharyngodon sp

Strongyloides cruzi

Acuariidae

Physaloptera sp.

Spirurinae

Oswaldocruzia brasiliensis

Oochoristica vanzolinii

Paradistomum parvissimum

Plagiorchis vicentei

Family Phyllodactylidae

Bogertia lutzae

Spauligodon oxkutzcabiensis

Gymnodactylus geckoides

Paradistomum rabusculum

Phyllodactylus angustidigitus

Spauligodon viracochai

\section{Phyllodactylus inaequalis}

Spauligodon oxkutzcabiensis

Phyllodactylus gerrhopygus

Spauligodon viracochai

\section{Phyllodactylus johnwrighti}

Oochoristica travassosi

Parapharyngodon sceleratus

Spauligodon oxkutzcabiensis

Phyllodactylus lepidopygus

Acuariidae
Phyllodactylus microphyllus

Spauligodon oxkutzcabiensis

Phyllodactylus reissii

Spauligodon oxkutzcabiensis

Phyllopezus pollicaris

Macdonaldius grassi

Parapharyngodon sceleratus

Thecadactylus solimoensis

Spauligodon oxkutzcabiensis

Physaloptera retusa

Physalopteroides venancioi

Ophiotaenia nicoleae

\section{Family Sphaerodactylidae}

Gonatodes humeralis

Skrjabinelazia galliardi

\section{Family Anguidae}

Diploglossus lessonae

Parapharyngodon verrucosus

Mesocoelium monas

\section{Ophiodes striatus}

Cyrtosomum sp.

Physaloptera retusa

\section{Family Teiidae}

\section{Ameiva ameiva}

Oligacanthorhynchus sp.

Centrorhynchus tumidulus

Capillaria (= Amphibiocapillaria) freitaslenti

Spinicauda spinicauda

Strongyluris oscari

Parapharyngodon alvarengai

Parapharyngodon largitor

Parapharyngodon sceleratus

Parapharyngodon sp.

Pharyngodon cesarpintoi

Pharyngodon travassosi

Chabirenia cayennensis

Piratuba scaffi

Physaloptera lutzi

Physaloptera retusa

Physaloptera sp.

Physalopteroides venancioi

Oochoristica ameivae

Oochoristica insulamargaritae

Paradistomum parvissimum 
Ameiva festiva

Physaloptera retusa

Cnemidophorus abaetensis

Physaloptera lutzi

Physaloptera retusa

\section{Cnemidophorus lemniscatus}

Physaloptera retusa

\section{Cnemidophorus littoralis}

Hexametra boddaertii

Parapharyngodon sceleratus

Physaloptera lutzPhysaloptera retusa

Physaloptera sp.

\section{Cnemidophorus nativo}

Skrjabinellazia intermedia

Subulura lacertilia

Parapharyngodon sp.

Physaloptera retusa

Physalopteroides venancioi

Oochoristica ameivae

\section{Cnemidophorus ocellifer}

Hexametra boddaertii

Physaloptera retusa

Physaloptera sp.

\section{Cnemidophorus sp.}

Pharyngodon cesarpintoi

\section{Dicrodon heterolepis}

Pharyngodon micrurus

Thubunaea parkeri

Oochoristica freitasi

\section{Dicrodon guttulatum}

Oochoristica freitasi

Oochoristica iguanae

Pharyngodon micrurus

Parapharyngodon moqueguensis

Parapharyngodon lagitor

Parapharyngodon sp.

Thelandros capacyupanquii

Physaloptera sp.

\section{Dicrodon holmbergi}

Thelandros capacyupanquii

\section{Dracaena guianensis}

Oswaldofilaria belemensis
Diaphanocephalus jacuruxi

Kentropyx altamazonica

Physaloptera retusa

Physalopteroides venancioi

\section{Kentropyx calcarata}

Kentropyxia sauria

Piratuba digiticauda

Piratuba shawi

Physaloptera retusa

Physalopteroides venancioi

Oswaldocruzia sp.

\section{Kentropyx pelviceps}

Dujardinascaris sp.

Parapharyngodon sceleratus

Physaloptera retusa

Physalopteroides venancioi

Ophiotaenia flava

\section{Tupinambis longilineus}

Physaloptera retusa

\section{Tupinambis merianae}

Cruzia travassosi

Physaloptera sp.

Diaphanocephalus galeatus

Spinicauda spinicauda

Oochoristica sp.

\section{Tupinambis rufescens}

Physaloptera retusa

Diaphanocephalus galeatus

Styphlodora condita

\section{Tupinambis teguixin}

Centrorhynchus tumidulus

Centrorhynchus sp.

Contracaecum sp.

Dujardinascaris sp.

Freitasascaris alata

Atractis cruciata

Spinicauda spinicauda

Cruzia fulleborni

Cruzia tentaculata

Oswaldofilaria petersi

Oswaldofilaria sp.

Physaloptera retusa

Physaloptera sp.

Physalopteroides venancioi

Diaphanocephalus diesingi 
Diaphanocephalus galeatus

Tejidotaenia appendiculata

Pulchrosomoides elegans

Paradistomum parvissimum

Dasymetra tupinambis

Family Gymnophthalmidae

Alopoglossus angulatus

Cosmocerca vrcibradici

Oswaldocruzia vitti

Mesocoelium monas

Alopoglossus atriventris

Cosmocerca vrcibradici

Physalopteroides venancioi

Oswaldocruzia vitti

Oochoristica sp.

Cercosaura argulus

Cosmocercidae

Acuariidae

Physaloptera sp.

Oswaldocruzia peruensis

\section{Cercosaura eigenmanni}

Cosmocerca vrcibradici

Oswaldocruzia vitti

Mesocoelium monas

\section{Cercosaura ocellata}

Physalopteroides venancioi

\section{Cercosaura oshaugnessyi}

Acanthocephalus saurius

Cosmocerca vrcibradici

Oswaldocruzia vitti

\section{Leposoma osvaldoi}

Brachycoelium salamandrae

\section{Neusticurus bicarinatus}

Falcaustra belemensis

Potamites ecpleopus

Falcaustra belemensis

Physaloptera retusa

Potamites strangulatus

Falcaustra belemensis

Family Scincidae

Mabuya agilis
Centrorhynchidae

Hexametra boddaertii

Strongyluris oscari

Parapharyngodon largitor

Parapharyngodon sceleratus

Acuariidae

Physaloptera retusa

Physaloptera sp.

Physalopteroides venancioi

Oochoristica ameivae

Paradistomum parvissimum

Trachylepis atlantica

Moaciria alvarengai

Parapharyngodon alvarengai

Mesocoelium monas

Spinicauda spinicauda

Platynosomum sp.

Oochoristica sp.

\section{Mabuya bistriata}

Parapharyngodon sceleratus

Oswaldofilaria spinosa

Piratuboides zeae

Physaloptera retusa

Physalopteroides venancioi

Mabuya caissara

Parapharyngodon sceleratus

Mabuya dorsivittata

Skrjabinodon spinulosus

Physaloptera retusa

Mabuya frenata

Hexametra boddaertii

Parapharyngodon sceleratus

Skrjabinodon heliocostai

Oswaldofilaria sp.

Oochoristica sp.

Mabuya macrorhyncha

Echinorhynchidae

Centrorhynchidae

Hexametra boddaertii

Acuariidae

Physaloptera sp.

Physalopteroides venancioi

Oochoristica ameivae

Pulchrosomoides elegans

Paradistomum parvissimum 


\section{FINAL REMARKS}

The literature on helminth parasites of lizards and amphisbaenians from South America is extensive, dating back to the 1920's with the studies by Lauro Travassos. However, the information is scattered, and reviews of helminth parasites are necessary because they provide a basis for a better understanding on parasitism in these groups for future research (180). The need of updated knowledge becomes clear considering the valuable information that parasites provide because it is synergistic with all the information about the natural history of the hosts (181).

Helminths are high diverse in vertebrates, but the species richness are poorly known $(182,183)$. For example, less than a half of nematode species are believed to be currently described (148). In this paper, a total of 644 records of helminths collected from lizards are reported. Since many species descriptions were made in the last decade, the diversity of helminth parasites of lizards from South America should be increased with further studies.

Of the 644 records in the present study, at least nine species of acantocephalans, 15 cestodes, 20 trematodes and 111 nematodes were reported, including records in which family or genus were not identified. From the 155 helminth species reported, one was recorded from Chile and French Guiana, three were from Colombia, three were from Uruguay, eight were from Bolivia, nine were from Surinam, 13 were from Paraguay, 12 were from Venezuela, 27 were from Ecuador, 17 were from Argentina, 39 were from Peru and 103 were from Brazil. Five species considered species inquirenda were also reported.

Lizards and amphisbaenians comprises approximately 420 genera and more than 3,300 species, most of which can be found in South America (184, 185). Many South American countries exhibit great lizard diversity, for example Argentina presents 167 species while Brazil has 240 species, but data presented herein includes only 114 lizard species, which indicates that much work has to be done $(186,187)$.

\section{ACKNOWLEDGEMENTS}

Both authors are thankful for the financial support of The State of São Paulo Research Foundation (FAPESP) and Robson W. Ávila is grateful for the grant provided by Coordination for the
Improvement of Higher Education Personnel (CAPES).

\section{COPYRIGHT \\ (C) CEVAP 2010}

\section{SUBMISSION STATUS}

Received: June 1, 2010.

Accepted: September 16, 2010.

Abstract published online: September 17, 2010.

Full paper published online: November 30, 2010.

\section{CONFLICTS OF INTEREST}

There is no conflict.

\section{FINANCIAL SOURCE}

FAPESP (processes n. 04/03628-1 and 06/59692-

5 ) and CAPES provided the financial grants.

\section{CORRESPONDENCE TO}

ROBSON WALDEMAR ÁVILA, Departamento de Parasitologia, Instituto de Biociências, UNESP, Distrito de Rubião Jr., s/n, CEP 18618-000, Botucatu, SP, Brazil. Email: robsonavila@gmail. com.

\section{REFERENCES}

1. Bursey CR, Goldberg SR, Parmelee JR. Gastrointestinal helminths from 13 species of lizards from Reserva Cuzco Amazónico, Peru. Comp Parasitol. 2005;72(1):50-68.

2. Goldberg SR, Bursey CR, Morando M. Metazoan endoparasites of 12 species of lizards from Argentina. Comp Parasitol. 2004;71(2):208-14.

3. Vrcibradic D, Anjos LA, Vicente JJ, Bursey CR. Helminth parasites of two sympatric lizards, Enyalius iheringii and E. perditus (Leiosauridae), from an Atlantic Rainforest area of southeastern Brazil. Acta Parasitol. 2008;53(2):222-5.

4. Travassos LP. Contribuições para o conhecimento da fauna helmintológica brasileira. IX. Sobre as espécies do gênero Spinicauda. Mem Inst Oswaldo Cruz. 1920;12:41-50.

5. Travassos LP. Pesquizas helminthologicas realizadas em Hamburgo. IX. Ensaio monographico da familia Cosmocercidae Travassos, 1925 (Nematoda). Mem Inst Oswaldo Cruz. 1931;25:237-98.

6. Travassos LP, Freitas JFT, Kohn A. Trematodeos do Brasil. Mem Inst Oswaldo Cruz 1969;67:1886.

7. Baker MR. Synopsis of the Nematoda parasitic in amphibians and reptiles. Mem Univ Newfoundland Occas Pap Biol. 1987;11:1-325. 
8. Vicente JJ, Rodrigues HO, Gomes DC, Pinto RM. Nematóides do Brasil. Parte III: Nematóides de répteis. Rev Bras Zool. 1993;10(1):19-168.

9. Lunaschi LI, Drago FB. Checklist of digenean parasites of amphibians and reptiles from Argentina. Zootaxa. 2007;1476:51-68.

10. Smales LR. Acanthocephala in amphibians (anura) and reptiles (Squamata) from Brazil and Paraguay with description of a new species. J Parasitol. 2007;93(2):392-8.

11. Yamaguti S. Systema helminthum. The nematodes of vertebrates. v. III. parts I and II. New York: Interscience Publishers; 1961.

12. Yamaguti S. Systema helminthum. The cestodes of vertebrates. v. II. New York: Interscience Publishers; 1959.

13. Schmidt GD. CRC Handbook of tapeworm identification. Florida: CRC Press; 1986. 675 p.

14. Yamaguti S. Systema helminthum. The digenetic trematodes of vertebrates. V. I. New York: Interscience Publishers; 1958.

15. Yamaguti S. Systema helminthum. Acanthocephala. v. V. New York: Interscience Publishers; 1963.

16. Lamas MF, Zaracho VH. Tropidurus torquatus. Endoparasites. Natural History. Herpetol Rev. 2006;37:474-5.

17. Vrcibradic D, Rocha CFD, Van Sluys M, Bursey CR. Mabuya macrorhyncha (NCN) Endoparasites. Herpetol Rev. 2001; 32:256.

18. BurseyCR, Goldberg SR. Cosmocerca vrcibradicin. sp. (Ascaridida: Cosmocercidae), Oswaldocruzia vitti n. sp. (Strongylida: Molineidae), and other helminths from Prionodactylus eigenmanni and Prionodactylus oshaughnessyi (Sauria: Gymnophthalmidae) from Brazil and Ecuador. J Parasitol. 2004;90(1):140-5.

19. Vicente JJ. Helmintos de Tropidurus (Lacertilia, Iguanidae) da Coleção Helmintológica do Instituto Oswaldo Cruz I. Trematoda, Cestoda, Acanthocephala, Linguatulida. Atas Soc. Biol. Rio de Janeiro 1978;19:71-78.

20. Vrcibradic D, Rocha CFD. Observations on the natural history of the lizard Mabuya macrorhyncha Hoge (Scincidae) in Queimada Grande island, São Paulo. Rev Bras Zool. 2005;22(4):1185-90.

21. Rodrigues HO. Contribuição ao estudo da fauna helmintológica de vertebrados de Nova Iguaçu, RJ. Atas Soc Biol. Rio de Janeiro.1986;26:27-8.

22. Anjos LA, Rocha CFD, Vrcibradic D, Vicente JJ. Helminths of the exotic lizard Hemidactylus mabouia from a rock outcrop area in southeastern Brazil. J Helminthol. 2005;79(4):307-13.

23. Vrcibradic D, Rocha CFD, Bursey CR, Vicente JJ. Helminth communities of two sympatric skinks (Mabuya agilis and Mabuya macrorhyncha) from two 'restinga' habitats in southeastern Brazil. J Helminthol. 2002;76(4):355-61.

24. Vrcibradic D, Vicente JJ, Bursey CR. Helminths infecting the lizard Enyalius bilineatus (Iguanidae, Leiosaurinae), from an Atlantic Rainforest area in Espirito Santo state, southeastern Brazil. Amphib-Reptil. 2007;28:166-169.

25. Neiva A, Cunha AM, Travassos LP. Contribuições parazitolojicas. Mem Inst Oswaldo Cruz.1914;6(3):180-91.

26. Travassos L. Contribuições para o conhecimento da fauna helminthologica brasileira. XX Revisão dos Acanthocephalos brasileiros. Parte II. Familia Echinorhynchidae Hamann, 1892, sub-fam. Centrorhynchinae Travassos, 1919. Mem Inst Oswaldo Cruz.1926;19(1):31-125.

27. RodriguesHO.Pseudocapillaria(Ichthyocapillaria) maricaensis n. sp. (Nematoda, Capillariidae) and remarks on the helminthological fauna of Liolaemus lutzae Mertens, 1938 (Lacertilia, Iguanidae). Mem Inst Oswaldo Cruz. 1992; 87:297-300.

28. Baylis HA. Some Roundworms and Flatworms from the West Indies and Surinam. I. Nematodes and Acanthocephala. J Linn Soc London.1947;41:394-405.

29. Lent H, Freitas JFT. Uma coleção de nematódeos de vertebrados, do Museu de Historia Natural de Montevideo. Mem Inst Oswaldo Cruz.1948;46(1):1-71.

30. Sprent JFA. Ascaridoid nematodes of amphibians and reptiles: Freitasascaris n.g. J Helminthol. 1983;57:283-90.

31. Dias EJR, Vrcibradic D, Rocha CFD. Endoparasites infecting two species of whiptail lizard (Cnemidophorus abaetensis and C. ocellifer; Teiidae) in a restinga habitat of northeastern Brazil. Herpetol J. 2005;15(2):133-7.

32. Ribas SC, Rocha CFD, Teixeira-Filho PF, Vicente JJ. Nematode infection in two sympatric lizards (Tropidurus torquatus and Ameiva ameiva) with different foraging tactics. AmphibReptil.1998;19(3):323-30.

33. Rocha CFD, Vrcibradic D. Nematode assemblages of some insular and continental lizard hosts of the genus Mabuya Fitzinger (Reptilia, Scincidae) along the eastern Brazilian coast. Rev Bras Zool. 2003;20(4):755-9.

34. Vrcibradic D, Rocha CFD, Ribas SC, Vicente JJ. Nematodes infecting the skink Mabuya frenata in Valinhos, São Paulo State, southeastern Brazil. Amphib-Reptil. 1999;20:333-9.

35. Vrcibradic D, Cunha-Barros M, Vicente JJ, Galdino CAC, Hatano FH, Van Sluys M, et al. Nematode infection patterns in four sympatric lizards from a restinga habitat (Jurubatiba) in Rio 
de Janeiro state, southeastern Brazil. AmphibReptil. 2000; 21(3):307-16.

36. Barus V. Coy Otero A. Systematic survey of nematodes parasitizing lizards (Sauria) in Cuba. Helminthol. 1969;10(3):329-46.

37. Vicente JJ. Redescrição de Atractis cruciata Linstow, 1902, em novo hospedeiro (Nematoda, Atractoidea). Atas Soc Biol Rio de Janeiro. 1966;10(2):41-3.

38. Bursey CR, Flanagan JP. Atractis marquezi n. sp. (Nematoda: Atractidae) and a revision of Atractis Dujardin, 1845, sensu Baker, 1987. J Parasitol. 2002;88:320-4.

39. McAllister CT, Bursey CR, Freed PS. Helminth parasites of selected amphibians and reptiles from the Republic of Ecuador. Comp Parasitol. 2010;77(1):52-66.

40. Goldberg SR, Bursey CR, Vitt LJ. Parasites of two lizard species, Anolis punctatus and Anolis transversalis (Squamata: Polychrotidae) from Brazil and Ecuador. Amphib-Reptil. 2006;27(4):575-9.

41. Ávila RW, Souza FL, Silva RJ. Helminths from seven species of lizards (Reptilia: Squamata) at the Cerrado of Mato Grosso do Sul State, Brazil. Comp Parasitol. 2010;77(1):67-71.

42. Adamson ML, Baccam D. Systematic position of the Atractidae sensu Chabaud (1978) (Nematoda: Cosmocercoidea): Maracaya belemensis n. sp. and Aplectana albae n. sp. from Amphisbaena alba in Brazil. Can J Zool. 1988;66:1857-64.

43. Boaumer S, Morand S. A new nematode (Nematoda: Cosmocercidae) from the lizard, Chamaeleo inturensis (Squamata: Chamaeleonidae) from the Democratic Republic of Congo. J Parasitol. 2006;92(2):346-9.

44. Ramallo GR, Bursey CR, Goldberg SR. A new species of Cosmocercidae (Ascaridida) in the worm lizard, Amphisbaena bolivica (Squamata: Amphisbaenidae), from Argentina. J Parasitol. 2008;94(6):1361-3.

45. Goldberg SR, Bursey CR. Cercosaura argulus endoparasites. Herpetol Rev. 2007;38(4):451.

46. Ramalho ACO, Silva RJ, Schwartz H, Péres AK. Helminths from an introduced species (Tupinambis merianae), and two endemic species (Trachylepis atlantica and Amphisbaena ridleyi) from Fernando de Noronha archipelago, Brazil. J Parasitol. 2009;95(4):1026-8.

47. Travassos LP. Aplecana raillieti, n. sp. C R Seanc Soc Biol. 1925;93:973-4.

48. Sousa BM, Lima SS, Oliveira A. Gastrointestinal helminth fauna of Enyalius perditus (Reptilia: Leiosauridae): Relation to host age and sex. J Parasitol. 2007; 93(1):211-3.

49. Freitas JFT, Vicente JJ. Nôvo nematódeo do gênero Cosmocerca Diesing, 1861, parasito de anfisbaenideo. Atas Soc Biol. Rio de Janeiro. 1966;10:109-11.

50. Bursey CR, Goldberg SR, Vitt LJ. New species of Allopharynx (Digenea: Plagiorchiidae) and other helminths in Uranoscodon superciliosus (Squamata: Tropiduridae) from Amazonian Brazil. J Parasitol. 2005;91(5):1395-8.

51. Goldberg SR, Bursey CR, Vitt LJ. Helminths of the brown-eared anole, Norops fuscoauratus (Squamata, Polychrotidae) from Brazil and Ecuador, South America. Phyllomedusa. 2006;5(1):83-6.

52. Goldberg SR, Bursey CR, Vitt LJ. Parasite communities of two lizard species, Alopoglossus angulatus and Alopoglossus atriventris, from Brazil and Ecuador. Herpetol J. 2007;17(4):26972.

53. Baker MR. Dollfusnema amphisbaenia n. gen., n. sp. (Nematoda: Cosmocercinae) from the lizard Leposternon phocaena (Amphisbaenia) of Brazil. Can J Zool. 1981;59(1):138-40.

54. Baker MR. Africana chabaudi n. sp. (Nematoda, Heterakidae) in a Brazilian Iguana. Bull Mus Nat His Nat Paris. 1981;3(3):783-7.

55. Ávila RW, Silva RJ. A new species of Africana (Nematoda: Heterakidae) from lizards of southern Amazon, Brazil. J Parasitol. 2009;95(5):1156-8.

56. Freitas JFT. Novo parasito de réptil da Ilha Fernando de Noronha: Moaciria alvarengai g. n., sp. n. (Nematoda, Subuluroidea). Rev Bras Biol. 1956;16:335-9.

57. Días-Ungría C. Notas sobre nematodes de reptiles en Venezuela. Rev Vet Venez. 1964;17(101):402.

58. Rodrigues HO, Feijó LMF. Redescrição de Spinicauda spinicauda (Olfers, 1919) Travassos, 1920 (Nematoda, Oxyuroidea). Atas Soc Biol Rio de Janeiro.1976; 18:59-63.

59. Travassos LP. Informações sobre a fauna helminthologica de Mato Grosso. Folha Med. 1923;4:58-60.

60. Pereira C. Os Oxyurata parasitos de Lacertilia do nordeste Brasileiro. Arch Inst Biol. 1935;6:5-27.

61. Bursey CR, Goldberg SR, Telford Jr SR. Strongyluris panamaensis n. sp. (Nematoda: Hheterakidae) and other helminths from the lizard, Anolis biporcatus (Sauria: Polychrotidae), from Panama. J Parasitol. 2003;89(1):118-23.

62. Vicente JJ. Helmintos de Tropidurus (Lacertilia, Iguanidae) da coleção helmintólogica do Instituto Oswaldo Cruz. II. Nematoda. Atas Soc Biol Rio de Janeiro. 1981;22:7-18.

63. Harvey MB, Gutberlet Jr RL. Lizards of the genus Tropidurus (Iguania: Tropiduridae) from the serrania de Huanchaca, Bolivia: New species, natural history, and a key to genus. 
Herpetologica. 1998; 54:493-520.

64. Alho CJR. Oxyurata de lagartos do Planalto Central. Sobre o gênero Strongyluris Mueller, 1894 com descrição de duas espécies novas. Rev Bras Biol. 1969;29:65-74.

65. Bursey CR, Goldberg SR. Helminths of Tropidurus guarani (Sauria: Tropiduridae) from Paraguay. Comp Parasitol. 2004;71(2):203-7.

66. Fontes AF, Vicente JJ, Kiefer MC, Van Sluys M. Parasitism by helminths in Eurolophosaurus nanuzae (Lacertilia: Tropiduridae) in an area of rocky outcrops in Minas Gerais state, southeastern Brazil. J Herpetol. 2003;37(4):736-41.

67. Kohn A, Pinto RM, Fernandes BMM. Contribuição ao conhecimento de Strongyluris oscari Travassos, 1923 (Nematoda, Subuluroidea). Mem Inst Oswaldo Cruz. 1973;71(3):219-25.

68. Sutton CA, Mordeglia C, Cruz F. Strongyluris oscari Travassos, 1923 (Nematoda, Heterakidae) en Tropidurus spinulosus (Squamata, Tropiduridae) Del Noroeste Argentino. Gayana Zool. 1998;62:171-5.

69. Roca V. Tropidurus melanopleurus (NCN) Parasites. Herpetol Rev. 1997; 28:204.

70. Khalil M, Vogelsang EG. Cruzia fulleborni, a new species of Nematoda from Tupinambis teguixin. Zentralbl Bakteriol I Originale. 1930;119(12):72-4.

71. Ruiz JM. Revisão do gênero Cruzia (Nematoda: Oxyuroidea) e estudo das espécies brasileiras [doctoral dissertation]. São Paulo (SP): Faculdade de Farmácia e Odontologia da USP; 1947. 105p.

72. Schuurmans-Stekhoven JH. Nematodos parasitarios del chaco Paraguayo y de Argentina del Museo de Estocolmo. Acta Zool Lilloana.1950;9:325-45.

73. Ávila RW, Cáceres NC, Ferreira VL, Silva RJ. Hoplocercus spinosus. Endoparasites. Herpetol Rev. 2008;39(1):88-9.

74. Chabaud AG. Skrjabinelazia Sypliaxov, 1930 (=Salobrella Freitas, 1940), genre de transition entre Cosmocercidés et Spirurides. Ann Parasitol Hum Comp. 1973; 48:329-34.

75. Chabaud AG, Bain O, Poinar GO. Skrjabinelazia galliardi (Nematoda, Seuratoidea): compléments morphologiques et cycle biologique. Ann Parasitol Hum Comp.1988;63(4):278-84.

76. Freitas JFT. Sobre um interessante nematódeo parasito de reptil (Spiruroidea). Mem Inst Oswaldo Cruz.1940;35(3):603-5.

77. Menezes VA, Vrcibradic D, Vicente JJ, Dutra GF, Rocha CFD. Helminths infecting the parthenogenetic whiptail lizard Cnemidophorus nativo in a restinga habitat of Bahia State, Brazil. J Helminthol. 2004;78(4):323-8.

78. Vicente JJ, Van Sluys M, Fontes AF, Kiefer MC.
Subulura lacertilia sp.n. (Nematoda, Subuluridae) parasitizing the Brazilian lizard Tropidurus nanuzae Rodrigues (Lacertilia, Tropiduridae). Rev Bras Zool. 2000;17(4):1065-8.

79. Araújo P. Um novo Oxyurideo Gynaecometra bahiensis n. gen. n. sp. (Nematoda: Oxyuroidea: Oxyuridae) encontrado em lacertílio. Mem Inst Butantan. 1976;40/41:251-7.

80. Tantaléan M. Nuevos registros de nemátodes parasitos de animales de vida silvestre en el Peru. Rev Peru Biol.1998;5(2):103-4.

81. Lopes SG, Silva LEM, Dantas EF, Almeida WO. Infecção por helmintos em três espécies de lagartos do Nordeste Brasileiro. Cad Cult Ciênc. 2006;1(1):47-51.

82. Arrojo L. Parásitos de animales silvestres en cautiverio en Lima, Perú. Rev Peru Biol. 2002;9(2):118-20.

83. Inglis WG, Diaz-Ungría C, Coles WJ. Nematodes de Venezuela. IV. Nematodes parásitos de vertebrados venezolanos, II. Acta Biol Venez. 1960;3:1-24.

84. Freitas JFT. Sobre os generos Thelandros Wedl, 1962 e Parapharyngodon Chatteuji, 1933, com descrição de Parapharyngodon alvarengai sp. n. (Nematoda, Oxyuroidea). Mem Inst Oswaldo Cruz. 1957;55(1):21-45.

85. Padilha TN, Duarte MJF. Ocorrência de Parapharyngodon alvarengai Freitas, 1957, em Ameiva ameiva (L.) no estado do Rio de Janeiro (Nematoda, Oxyuroidea). Atas Soc. Biol. Rio de Janeiro. 1979; 20:21-22.

86. Ramallo GR, Bursey CR, Goldberg SR. Parapharyngodon riojensis n. sp. (Nematoda: Pharyngodonidae) from the lizard Phymaturus punae (Squamata: Iguania: Liolaemidae) from northwestern Argentina. J Parasitol. 2002;88(5):979-82.

87. Bursey CR, Brooks DR. Parapharyngodon duniae n. sp. (Nematoda: Pharyngodonidae) in Phrynohyas venulosa (Anura: Hylidae) from the Area de Conservación Guanacaste, Guanacaste, Costa Rica. J Parasitol. 2004;90(1):137-9.

88. Bursey CR, Goldberg SR. Two new species of Pharyngodonidae (Nematoda: Oxyuroidea) and other Nematodes in Agama caudospina (Squamata: Agamidae) from Kenya, Africa. J Parasitol. 2005;91(3):591-9.

89. Calisaya JL, Cordova E. Tres nuevas espécies de Parapharyngodon (Nematoda, Oxiuroidea) parásitas de Tropidurus peruvianus del sur del Perú. Rebiol. 1997;17(1-4):45-54.

90. Morales E, Sarmiento L, Sánchez L, Florindéz D, Lamas G. Material tipo de helmintos en el Museo de Historia Natural, Universidad Nacional Mayor de San Marcos, (MUSM), Lima, Perú. Rev Peru 
Biología. 2005;12(3):463-72.

91. Rodrigues HO, Pinto RM. Ocorrência de dois faringodonídeos em Ameiva ameiva (L.) no estado da Guanabara (Nematoda, Oxyuroidea). Atas Soc Biol Rio de Janeiro.1967;11(3):123-5.

92. Vásquez PTS. Helmintos parásitos de Dicrodon guttulatum, Dúmeril y Bibron, 1893 (SAURIA: TEIIDAE) de la costa del Perú [dissertation]. Lima, Peru: Facultad Ciencias Biológicas, Universidad Nacional Mayor de San Marcos; 2008. 63p.

93. Alho CJR, Rodrigues HO. Sobre novo hospedeiro de Parapharyngodon sceleratus (Travassos, 1923) Freitas, 1957, com redescrição da espécie (Nematoda, Oxyuroidea). Atas Soc Biol Rio de Janeiro.1963; 7:4-6.

94. Ribas SC, Rocha CFD, Teixeira-Filho PF, Vicente JJ. Helminths (Nematoda) of the lizard Cnemidophorus ocellifer (Sauria: Teiidae): assessing the effect of rainfall, body size and sex in the nematode infection rates. Ciênc Cult. 1995;47(1-2):88-91.

95. Van Sluys M, Rocha CFD, Ribas SC. Nematodes infecting the lizard Tropidurus itambere in southeastern Brazil. AmphibReptilia.1994;15(4):405-8.

96. Van Sluys M, Rocha CFD, Bergallo HG, Vrcibradic D, Ribas SC. Nematode infection in three sympatric lizards in an isolated fragment of restinga habitat in southeastern Brazil. AmphibReptil.1997;18(4):442-6.

97. Goldberg SR, Bursey CR. Helminths from four species of Phyllodactylus (Squamata: Gekkonidae) from Peru. Comp Parasitol. 2010;77(1):91-3.

98. Freitas JFT. Sobre um novo nematódeo de reptil da Bolivia: Parapharyngodon senisfaciecauduts sp. n. (Nematoda, Oxyuroidea). Rev Bras Biol. 1957;17:451-4.

99. Freitas JFT, Dobbin Jr, JE. Nova espécie do gênero Parapharyngodon Chatterji, 1933 (Nematoda, Oxyuroidea). An Soc Biol Pernambuco. 1959;16:23-33.

100. Rodrigues HO, Rodrigues SS, Faria Z. Contribution to the knowledge of the helminthological fauna of vertebrates of Maricá, Rio de Janeiro State, Brazil. Mem Inst Oswaldo Cruz.1990;85(1):115-6.

101. Zaracho VH, Lamas MF. Tropidurus etheridgei. Endoparasites. Natural History. Herpetol Rev. 2006; 37:473.

102. Cruz FB, Silva S, Scrocchi GJ. Ecology of the lizard Tropidurus etheridgei (Squamata: Tropiduridae) from the dry Chaco of Salta, Argentina. Herpetol Nat Hist. 1998;6(1):23-31.

103. Rocha CFD. Nematode parasites of the Brazilian sand lizard, Liolaemus lutzae. Amphib-
Reptil.1995;16(4):412-5.

104. Alho CJR, Moura VP. Oxyurata de lagartos do Planalto Central. Ocorrência de Pharyngodon cesarpintoi Pereira, 1935 (Nematoda, Subuluroidea). Atas Soc Biol Rio de Janeiro.1970;13(1-2):67-9.

105. Freitas JFT, Ibáñez N. Fauna helmintológica del Perú - Notas sobre "Pharyngodonidae" Travassos, 1920 y descripción de Pharyngodon micrurus sp. n. Rev. Universitaria 1963; 23 y 24.

106. Bursey CR, Goldberg SR. New species of Skrjabinodon (Nematoda: Pharyngodonidae) in Uracentron flaviceps (Squamata: Iguanidae) from Ecuador and Peru. J Parasitol. 2007;93(4):866-9.

107. Vicente JJ, Vrcibradic D, Muniz-Pereira LC, Pinto PM. Skrjabinodon heliocostai sp. n. (Nematoda, Pharyngodonidae) parasitizing Mabauya frenata (Cope) (Lacertilia, Scincidae) in Brazil and the reallocation of Skrjabinodon capacyupanquii (Freitas, Vicente \& Ibanez) in the genus Thelandros Wedl. Rev Bras Zool. 2000; 17:361-367.

108. Rocha CFD, Vrcibradic D, Vicente JJ, CunhaBarros M. Helminths infecting Mabuya dorsivittata (Lacertilia, Scincidae) from a highaltitude habitat in Itatiaia National Park, Rio de Janeiro State, southeastern Brazil. Braz J Biol. 2003;63(1):129-32.

109. Vicente JJ, Vrcibradic D, Rocha CFD, Pinto RM. Description of Skrjabinodon spinosulus sp. $\mathrm{n}$. (Nematoda, Oxyuroidea, Pharyngodonidae) from the Brazilian lizard Mabuya dorsivittata Cope, 1862 (Scincidae). Rev Bras Zool. 2002;19(1):15762.

110. Ramallo GR, BurseyCR, GoldbergSR.Spauligodon loboi n. sp. (Nematoda: Pharyngodonidae) parasite of Liolaemus spp. (Iguania: Liolaemidae) from northwestern Argentina. J Parasitol. 2002;88(4):370-4.

111. Vicente JJ, Ibañez HN. Nova espécie do gênero Parathelandros Baylis, 1930 (Nematoda, Oxyuroidea). Atas Soc Biol Rio de Janeiro.1968;11:185-7.

112. Goldberg SR, Muñoz G, Garcias F, Bursey CR. Liolaemus lemniscatus (wreath tree iguana), Liolaemustenuis (thin tree iguana). Endoparasites. Herpetol Rev. 2001;32:41.

113. Bergmann PJ, Russell AP. Systematics and biogeography of the widespread Neotropical gekkonid genus Thecadactylus (Squamata), with the description of a new cryptic species. Zool J Linnean Soc. 2007;149:339-70.

114. Goldberg SR, Bursey CR. Phyllodactylus reissi Endoparasites. Herpetol Rev. 2004; 35:395.

115. Ávila RW, Anjos LA, Gonçalves U, Freire EMX, Almeida WO, Silva RJ. Nematode infection in the lizard Bogertia lutzae (Loveridge, 1941) 
from the Atlantic forest in northeastern Brazil. J Helminthol. 2010; 89(2):199-201.

116. Freitas JFT, Vicente JJ, Ibáñez N. Fauna helmintológica do Peru: Novo nematódeo do gênero Parathelandros Baylis, 1930 (Nematoda, Oxyuroidea). Atas Soc Biol Rio de Janeiro.1968;12:33-35.

117. Pérez ZJ, Balta K, Salizar P, Sánchez L. Nematofauna de tres especies de lagartijas (Sauria: Tropiduridae y Gekkonidae) de la Reserva Nacional de Paracas, Ica, Perú. Rev Peru Biol. 2007;14(1):43-5.

118. Freitas JFT, Vicente JJ, Ibáñez N. Fauna Helmintológica del Perú: Parathelandros capacyupanquii sp. n., parasito de Dicrodon holmbergiSchmidt, 1957(Nematoda, Oxyuroidea). Atas Soc Biol Rio de Janeiro.1968;11:217-9.

119. Lhermitte-Vallarino L, Bain O, Deharo E, Bertani $\mathrm{S}$, Voza $\mathrm{T}$, Attout $\mathrm{T}$, et al. A new rhabdiasid nematode, Chabirenia cayennensis n. g., n. sp., parasitic in the glands of the buccal mucosa of a South American saurian. Syst Parasitol. 2005;62(2):151-60.

120. Muniz-Pereira LC, Vieira FM, Luque JL. Checklist of helminth parasites of threatened vertebrate species from Brazil. Zootaxa. 2009;2123:1-45.

121. Almeida WO, Ribeiro SC, Santana GG, Vieira WLS, Anjos LA, Sales DL. Lung infection rates in two sympatric Tropiduridae lizard species by pentastomids and nematodes in northeastern Brazil. Braz J Biol. 2009;69(3):963-7.

122. Rodrigues HO. Sobre nova espécie do gênero Strongyloides. Atas Soc Biol Rio de Janeiro.1968;12:31-32.

123. Freitas JFT, Rodrigues HO. Nótula helmintológica: sobre o material original de Filaria multipapilla Molin, 1858 atualmente existente no Museu de Viena. Atas Soc Biol Rio de Janeiro.1964;8:37-8.

124. Lent H, Freitas JFT. Sobre os filarídeos parasitas de lacertídeos neotropicos. Rev Bras Biol. 1941;1(4):383-6.

125. Vicente JJ, Jardim CR. Filarídeos da Coleção helmintológica do Instituto Oswaldo Cruz. I. Peixes, anfíbios e répteis. Atas Soc Biol Rio de Janeiro. 1980; 21:47-57.

126. McAllister CT, Bursey CR, Freed PS. Helminth parasites (Cestoda: Nematoda) of selected herpetofauna from Paraguay. J Parasitol. 2010;96(1):222-4.

127. Bain O. Description de nouvelles filaires Oswaldofilariinae de lezards sud-americains; hypothese sur l'evolution des filaires de reptiles. Bull Mus natn Hist nat Paris, Zool.1974;138(108):169-200.

128. Freitas JFT, Lent H. Sobre Oswaldofilaria brevicaudata (Rhodian and Vuylssteke, 1937) n. comb. (Nematoda; Filarioidea). Mem Inst Oswaldo Cruz. 1937;32:439-42.

129. Días-Ungría C. Helmintos parásitos de vertebrados en el estado Zulia (Venezuela) algunas especies nuevas para Venezuela. Vet Trop.1978;3:15-37.

130. Silva RJ, Kohlsdorf T. Tropidurus hispidus Spix 1825 (Sauria, Tropiduridae): a new host for Oswaldofilaria petersi Bain \& Sulahian 1974 (Nematoda, Onchocercidae). Arq Bras Med Vet Zootec. 2003;55(3):377-9.

131. Ávila RW, Silva RJ. Helminths of the teiid lizard Kentropyx calcarata (Squamata) from an Amazonian site in western Brazil. J Helminthol. 2009;83(3):267-9.

132. Ramallo GR, DíazF. Physaloptera lutzi (Nematoda, Physalopteridae) parasite de Liolaemus (Iguania, Tropiduridae) del noroeste Argentino. Bol Chil Parasitol. 1998;53(1-2):19-22.

133. Cristofaro R, Guimarães JF, Rodrigues HO. Alguns nematódeos de Tropidurus torquatus (Wied) e Ameiva ameiva (L.)_Fauna Helmintológica de Salvador, Bahia. Atas Soc Biol Rio de Janeiro. 1976;18:65-70.

134. Diaz-Ungria C, Gallardo MF. Nematodes de reptiles Venezolanos, con descripción de varias especies nuevas. Bol Soc Vem Cienc Nat. 1968; 27(113-114):550-70.

135. Vicente JJ, Santos E. Ocorrência de Physaloptera retusa Rudolphi, 1819 em novo hospedeiro (Nematoda, Physalopteridae). Atas Soc Biol Rio de Janeiro. 1967; 11:75-7.

136. Goldberg SR, Bursey CR, Caldwell JP, Vitt LJ, Costa GC. Gastrointestinal Helminths from six species of frogs and three species of lizards, sympatric in Pará State, Brazil. Comp Parasitol. 2007;74:327-42.

137. Goldberg SR, Bursey CR. Uracentron flaviceps. Endoparasites. Herpetol Rev. 2007;38(1):85.

138. Noronha D, Bragança R, Vicente JJ, Pereira LCM. Coleções particulares incorporadas à coleção helmintológica do Instituto Oswaldo Cruz (CHIOC). I: Coleção do Instituto Pasteur de São Paulo. Rev Bras Zool. 2004;21(2):303-5.

139. Goldberg SR, Bursey CR. Ameiva festiva Endoparasites. Herpetol Rev. 2009;40(1):81-2.

140. Prieto AS. Note on parasites of the tropical lizard Tropidurus hispidus. J Herpetol.1980;14(2):190-2.

141. Caballero E, Vogelsang EG. Fauna helmintológica venezolana. I. Ochetosoma miladelarocai n. sp. de Bothrops atrox L. y hallazgo de Physaloptera retusa (Rud., 1819) en Cnemidophorus lemniscatus lemniscatus. Rev Med Vet Parasitol.1947;6:53-62.

142. Ávila RW, Anjos LA, Silva RJ, Costa HC, São Pedro VA, Feio RN. Tupinambis longilineus. Endoparasites. Herpetol Rev. 2010;41(1):87-8.

143. Silva AS, Zanette RA, Tochetto C, Oliveira 
CB, Soares JB, Otto MA, et al. Parasitismo por Physaloptera sp., Kalicephalus sp. e Cryptosporidium sp. em lagarto (Tupinambis teguixin) no Rio Grande do Sul, Brasil. Zoociências. 2008;10(3):269-72.

144. de Fabio SP, Rolas FJT. Contribuição ao conhecimento de Thubuneae dactyluris Karve, 1938 (Nematoda, Spiruroidea). Mem Inst Oswaldo Cruz. 1974; 72(3-4):283-290.

145. Baylis HA. On a new Species of the Nematode Genus Thubunaea. Ann Mag Nat Hist.1926;9(18):361-4.

146. Rodrigues HO. Estudo da fauna helmintologica de Hemidactylus mabouia (M. de J.) no Estado da Guanabara. Atas Soc Biol Rio de Janeiro. 1970;12:15-23.

147. Travassos LP, Freitas JFT. Relatório da terceira excursão a zona da estrada de Ferro Noroeste do Brasil realizada em fevereiro e março de 1940. Mem Inst Oswaldo Cruz. 1941;35(3):607-96.

148. Anderson RC. (2000) Nematode Parasites of Vertebrates. Their Development and Transmission. Wallingford, Oxon, U.K.: CAB International; 2000. 650 pp.

149. Freitas JFT, Lent H. Pesquisas helmintológicas realizadas no estado do Pará. V. gênero Diaphanocephalus Diesing, 1851 (Nematoda: Strongyloidea). Mem Inst Oswaldo Cruz. 1938;33(3):423-32.

150. Spinelli CM, Fiorito LE, Stiebel C. Alteraciones histologicas en el intestino delgado en Tupinambis rufescens (Sauria, Teiidae) causadas por Diaphanocephalus galeatus (Nematoda, Diaphanocephalidae). Cuad Herpetol. 1992;7(6):38-40.

151. Alho CJR. Sobre uma espécie nova do gênero Diaphanocephalus Diesing, 1851, parasita de lagarto da Amazônia (Dracaena guianensis Daudin) (Nematoda, Strongyloidea). Bol Mus Par Emilio Goeldi Zool. 1965;59:1-5.

152. Travassos LP, Freitas JFT, Mendonça JM. Relatório da excursão do Instituto Oswaldo Cruz ao Parque de Reserva e Refúgio Sooretama, no estado do Espírito Santo, em outubro de 1963. Bol Mus Biol Mello-Leitão.1964;23(1):1-26.

153. Baker MR. On two new nematode parasites (Trichostrongyloidea: Molineidae) from amphibians and reptiles. Proc Helm Soc Wash. 1982;49:252-7.

154. Ben Slimane B, Durette-Desset MC. Four new species of Oswaldocruzia (Nematoda: Trichostrongylina, Molineoidea) parasitizing amphibians and lizards from Ecuador. Mem Inst Oswaldo Cruz. 1996;91(3):317-28.

155. Durette-Desset MC, Anjos LA, Vrcibradic D. Three new species of the genus Oswaldocruzia
Travassos, 1917 (Nematoda, Trichostrongylina, Molineoidea) parasites of Enyalius spp. (Iguanidae) from Brazil. Parasite. 2006;13(2):11525.

156. Lent H, Freitas JFT. Sobre uma nova espécie do gênero Oswaldocruzia Travassos, 1917. Mem Inst Oswaldo Cruz. 1935;30(3):379-86.

157. Ben Slimane B, Verhaagh M, Durette-Desset MC. Oswaldocruzia peruensis n. sp. (Nematoda: Trichostrongylina) parasite d'un Iguanidae du Pérou. Bull Mus Nat Hist Nat Paris. 1995;17:7782.

158. Freitas JFT. Nota sobre a fauna helmintológica de répteis brasileiros. Rev Bras Biol. 1955;15(3):27984.

159. Vicente JJ, Santos E. Contribuição ao conhecimento de Oochoristica fuhrmanni Hughes, 1940. Atas Soc Biol Rio de Janeiro. 1971;15:29-31.

160. Rego AA. Contribuição ao conhecimento dos Cestóides do Brasil. I-Cestóides de peixes, anfíbios e répteis. Atas Soc Biol Rio de Janeiro.1973;16(2):97-129.

161. Pinto RM, Gomes DC. Contribuição ao conhecimento da fauna helmintológica da região Amazônica - Cestódeos. Mem Inst Oswaldo Cruz. 1976; 74(1):53-64.

162. Rego AA, Rodrigues HO. Sobre duas Oochoristica parasitas de lacertílios (Cestoda, Cyclophillidea). Rev Bras Biol. 1965; 25(1):59-65.

163. Bursey CR, Goldberg SR. Oochoristica maccoyi n. sp. (Cestoda: Linstowiidae) from Anolis gingivinus (Sauria: Polychrotidae) collected in Anguilla, Lesser Antilles. Caribbean J Sci. 1996;32(4):3904.

164. Arizmendi-Espinosa MA, García-Prieto L, Guillén-Hernández S. A new species of Oochoristica (Eucestoda: Cyclophyllidea) parasite of Ctenosaura pectinata (Reptilia: Iguanidae) from Oaxaca, Mexico. J Parasitol. 2005; 91(1):99101.

165. Bursey CR, Goldberg SR, Telford Jr SR. Gastrointestinal helminths of 14 species of lizards from Panama with descriptions of five new species. Comp Parasitol. 2007;74(4):108-40.

166. Guillén-Hernández S, Garcia-Prieto L, Arizmendi-Espinosa MA. A new species of Oochoristica (Eucestoda: Cyclophyllidea) parasite of Ctenosaura oaxacana (Reptilia: Iguanidae) from México. J Parasitol. 2007;93(5):1136-9.

167. Rego AA, Ibañez HN. Duas novas espécies de Oochoristica, parasitas de lagartixas do Peru (Cestoda, Anoplocephalidae). Mem Inst Oswaldo Cruz.1965;63:67-73.

168. Lopez-Neyra CR, Diaz-Ungria C. Cestodes de Venezuela.- III. Sobre unos cestodes intestinales 
de reptiles y mamiferos venezolanos. Mem Soc Cienc Nat. La Salle.1957;17:28-63.

169. Rego AA. Sobre alguns cestódeos parasitos de Répteis. Rev Bras Biol. 1967;27(2):181-7.

170. Coquille SC, De Chambrier A. Cairaella henrii n. gen., n. sp., a parasite of Norops trachyderma (Polychrotidae) and Ophiotaenia nicolae n. sp. (Eucestoda: Proteocephalidea), a parasite of Thecadactylus rapicauda (Gekkonidae). Folia Parasitol. 2008; 55:197-206.

171. Rego AA, Chambrier A. Redescription of Tejidotaenia appendiculata (Baylis, 1947) (Cestoda: Proteocephalidea), a parasite of Tupinambis teguixin (Sauria: Teiidae) from South America. Mem Inst Oswaldo Cruz. 2000; 95(2):161-5.

172. Hughes RC, Higginbotham JW, Clary JW. The trematodes of reptiles-part 1. Am Midl Nat. 1942;27:109-34.

173. Freitas JFT, Lent H. Sobre um novo trematódeo parasita de Iguana tuberculata (Laur.). Mem Inst Oswaldo Cruz.1937;32(1):535-8.

174. Travassos LP. Informações sobre a fauna helmintológica de Mato Grosso. Fol Med. 1922;3:187-190.

175. Travassos LP. Fauna helminthologica de Mato Grosso (Trematódeos - $1^{\text {a }}$ Parte). Mem Inst Oswaldo Cruz. 1928; 21(2):309-41.

176. Travassos LP. Contribuição para a sistemática dos Dicrocoelinae Looss, 1899. Arc Esc Sup Agric Med Vet. 1919; 3:7-24.

177. Travassos LP. Relatório da excursão do Instituto Oswaldo Cruz ao município de Santa Teresa, no estado do Espírito Santo, em Agosto e Setembro de 1943. Mem Inst Oswaldo Cruz. 1944; 40(2):121-8.

178. Nasir P, Díaz MT. Flukes from Venezuelan reptiles with observations on intraspecific variations. Riv Parassitol. 1971;32(4):231-48.
179. Rodrigues HO. Plagiorchis vicentei n. sp.(Trematoda, Plagiorchiidae) a new trematode from Hemidactylus mabouia (Moreau de Jonne`s) (Lacertilia,Gekkonidae). Rev Bras Zool. 1994;11(4):669-72.

180. Muzzall PM. Parasites of amphibians and reptiles from Michigan: A review of the literature 1916-2003. Michigan Dept. Nat. Res., Fisheries Research Report 2005; 2077:1-30.

181. Rodríguez-Ortiz B, García-Prieto L, Pérez-Ponce de León B. Checklist of the helminth parasites of vertebrates in Costa Rica. Rev Biol Trop. 2004; 52(2):343-54.

182. Hugot JP, Baujard P, Morand S. Biodiversity in helminths and nematodes as a field of study: an overview. Nematol. 2001;3(3):199-208.

183. Gregory RD, Keymer AE, Harvey PH. Helminth parasite richness among vertebrates. Biodiv Conserv. 1996;5(3):985-97.

184. Pough FH, Andrews RM, Cadle JE, Crump ML, Savitzky AH, Wells KD. Herpetology. Second edition. New Jersey: Prentice-Hall; 2001. 612p.

185. Pianka ER, Vitt LJ. Lizards: Windows to the evolution of diversity. Berkeley: University of California Press; 2003. 333pp.

186. Lavilla E, Richard E, Scrocchi G. Categorización de los anfibios y reptiles de la república Argentina. San Miguel de Tucumán: Asociación Herpetológica Argentina; 2000. 97 p.

187. Bérnils RS. Brazilian reptiles - List of species [Internet]. Curitiba: Sociedade Brasileira de Herpetologia; c2004-2010 [updated 2010 March; cited 2010 April 6]. Available from: http://www. sbherpetologia.org.br/. 